\title{
Sparse-grid finite-volume multigrid for 3D-problems
}

\author{
P.W. Hemker \\ Centrum voor Wiskunde en Informatica, P.O. Box 94079, 1090 GB Amsterdam, The Netherlands
}

\begin{abstract}
We introduce a multigrid algorithm for the solution of a second order elliptic equation in three dimensions. For the approximation of the solution we use a partially ordered hierarchy of finite-volume discretisations. We show that there is a relation with semicoarsening and approximation by more-dimensional Haar wavelets. By taking a proper subset of all possible meshes in the hierarchy, a sparse grid finite-volume discretisation can be constructed.

The multigrid algorithm consists of a simple damped point-Jacobi relaxation as the smoothing procedure, while the coarse grid correction is made by interpolation from several coarser grid levels.

The combination of sparse grids and multigrid with semi-coarsening leads to a relatively small number of degrees of freedom, $N$, to obtain an accurate approximation, together with an $\mathcal{O}(N)$ method for the solution. The algorithm is symmetric with respect to the three coordinate directions and it is fit for combination with adaptive techniques.

To analyse the convergence of the multigrid algorithm we develop the necessary Fourier analysis tools. All techniques, designed for 3D-problems, can also be applied for the 2D case, and - for simplicity - we apply the tools to study the convergence behaviour for the anisotropic Poisson equation for this $2 \mathrm{D}$ case.
\end{abstract}

Keywords: Sparse grids, multigrid, finite volume, discrete Fourier transform, wavelets.

AMS subject classification: $65 \mathrm{~N} 55,65 \mathrm{~N} 22,65 \mathrm{~T} 20$.

\section{Introduction}

In this paper we describe the approximation of a function on a finite-volume sparse grid, and a multigrid algorithm for the solution of partial differential equations in three dimensions. The algorithm is intended for the solution of flow problems described by conservation laws, and therefore finite volumes are a natural choice for the discretisation. But to introduce the main principles, we will restrict the treatment here to second order elliptic equations, and in particular to the anisotropic Poisson equation.

In contrast to the usual multigrid approach, we do not use a sequentially ordered set of discretisations on different meshes, but we use a partially ordered hierarchy of "semi-coarsened" grids as proposed e.g. by Mulder [6,7] and Naik-VanRosendale [8] or Zenger et al. [3,9]. As indicated in [9], adaptive "sparse-grid" discretisations

(C) J.C. Baltzer AG, Science Publishers 
can be constructed by taking a suitable subset of all possible discretisations in such a hierarchy. However, in contrast to the sparse grid approximation proposed in $[3,9]$, we base our approximation on finite volumes rather than on finite elements.

The multigrid algorithm consists of damped Jacobi relaxation as a smoothing procedure and a coarse grid correction constructed by extrapolation from simultaneous corrections on several coarser grid levels.

The algorithm is completely symmetric with respect to the three coordinate directions and it is suitable for combination with adaptive techniques. A description of a data structure to implement such adaptive three-dimensional algorithms is given elsewhere [5].

\section{Finite-volume sparse grids}

In this section we introduce finite-volume sparse grids. We show the relation between the approximation by Haar wavelets (when this notion is extended to more dimensions) and the sparse-grid approximation. For the theory of wavelets, multiresolution analysis (MRA) etc. we refer to Daubechies [2].

\subsection{The more-dimensional MRA}

A multidimensional multiresolution analysis of $L^{2}(\Omega), \Omega=\mathbb{R}^{3}$, is a partially ordered set of closed linear subspaces

with the properties:

$$
\left\{V_{\mathbf{n}} V_{\mathbf{n}} \subset L^{2}(\Omega)\right\}_{\mathbf{n} \in \mathbb{Z}^{3}}
$$

$$
\begin{gathered}
\bigcap_{\mathbf{n}} V_{\mathbf{n}}=\{0\} ; \bigcup_{\mathbf{n}} V_{\mathbf{n}} \subset_{\text {dense }} L^{2}(\Omega) ; \\
f(\boldsymbol{x}) \in V_{\mathbf{n}} \Leftrightarrow f\left(2^{\mathbf{m}} \boldsymbol{x}\right) \in V_{\mathbf{n}+\mathbf{m}} \quad \forall \mathbf{n} \in \mathbb{Z}^{3}, \mathbf{m} \in \mathbf{E} ; \\
f(\boldsymbol{x}) \in V_{\mathbf{n}} \Leftrightarrow f\left(\boldsymbol{x}-2^{-\mathbf{n}} \boldsymbol{k}\right) \in V_{\mathbf{n}} \quad \forall \boldsymbol{k} \in \mathbb{Z}^{3}, \mathbf{n} \in \mathbf{E} ; \\
\exists \phi \in V_{\mathbf{o}}:\{\phi(\boldsymbol{x}-\boldsymbol{k})\}_{\boldsymbol{k} \in \mathbb{Z}^{3}} \text { is a Riesz basis for } V_{\mathbf{0}} .
\end{gathered}
$$

Here $\mathbf{n}=\left(n_{1}, n_{2}, n_{3}\right) \in \mathbb{Z}^{3}$, and we denote $|\mathbf{n}|=n_{1}+n_{2}+n_{3} ; 2^{\mathbf{n}}=\left(2^{n_{1}}, 2^{n_{2}}, 2^{n_{3}}\right)$. We also use the notation $\mathbf{o}=(0,0,0) \in \mathbb{N}^{3} ; \boldsymbol{x}=\left(x_{1}, x_{2}, x_{3}\right) \in \mathbb{R}^{3} ; 2^{\mathrm{n}} \boldsymbol{x}=\left(2_{1}^{n} x_{1}\right.$, $\left.2^{n_{2}} x_{2}, 2^{n_{3}} x_{3}\right)$. Further, we introduce in $\mathbb{N}^{3}$ the unit vectors $\mathbf{e}_{k}, k=1,2,3$, as follows: $\mathbf{e}_{1}(1,0,0) ; \mathbf{e}_{2}=(0,1,0) ; \mathbf{e}_{3}=(0,0,1)$, and we use $\mathbf{e}=(1,1,1)$. Finally we define $\mathbf{E}=\left\{\mathbf{e}_{1}, \mathbf{e}_{2}, \mathbf{e}_{3}\right\}$. Although we are particularly interested in the threedimensional case, generalisation to a different number of space dimensions is straightforward. The function $\phi(x)$ in (1.4) is called the scaling function of the multiresolution analysis.

\subsection{Piecewise constant function spaces}

Let either $\Omega=\mathbb{R}^{3}$ be the three-dimensional Euclidean space, or let 
$\Omega=(0,1)^{3} \subset \mathbb{R}^{3}$ be the open unit cube. For any $\mathbf{n} \in \mathbb{Z}^{3}$ we introduce the function space $V_{\mathrm{n}}$, i.e. the space of piecewise constant functions on a uniform grid with meshsize $\boldsymbol{h}=\left(2^{-n_{1}}, 2^{-n_{2}}, 2^{-n_{3}}\right)$. These grids are uniformly spaced in each of the three coordinate directions, but possibly with a different meshsize in the different directions. The volume of these cells is denoted by $h^{3}=2^{-|\mathbf{n}|}$. The functions in $V_{\mathbf{n}}$ are all constant in each cell

$$
\Omega_{\mathbf{n}, \boldsymbol{k}}=\left[k_{1} 2^{-n_{1}},\left(k_{1}+1\right) 2^{-n_{1}}\right] \times\left[k_{2} 2^{-n_{2}},\left(k_{2}+1\right) 2^{-n_{2}}\right] \times\left[k_{3} 2^{-n_{3}},\left(k_{3}+1\right) 2^{-n_{3}}\right],
$$

and this family of cells forms the grid $\Omega_{\mathbf{n}}=\left\{\Omega_{\mathbf{n}, k} \mid \Omega_{\mathbf{n}, k} \subset \Omega, k \in \mathbb{Z}^{3}\right\}$. The family of cell centres or cell nodes is denoted by $\Omega_{\mathbf{n}}^{*}=\left\{z_{\mathbf{n}, k} \mid \mathbf{z}_{\mathbf{n}, k}=(\boldsymbol{k}+\mathbf{e} / 2) 2^{-\mathbf{n}} ; \boldsymbol{k} \in \mathbb{Z}^{3}\right.$; $\left.\mathbf{z}_{\mathbf{n}, k} \in \bar{\Omega}\right\}$. The number of these nodes is equal to the dimension of $V_{\mathbf{n}}$.

Apparently, all grids are identified by a triple $\mathbf{n}$; the number $|\mathbf{n}|$ is called the level of the grid $\mathbf{n}$. Notice that - different from a classical multigrid - here and later in our multigrid algorithm, there is a clear distinction between the grid-identification index $\mathbf{n}$ and the level number $|\mathbf{n}|$.

Because

$$
V_{\mathbf{n}} \subset V_{\mathbf{n}+\mathbf{e}_{j}}, \quad \text { for } j=1,2,3,
$$

we see, for construction, that nesting relations exist between spaces $V_{\mathrm{n}}$ and that the nesting provides a partial ordering ${ }^{1}$ :

$$
V_{\mathbf{n}} \subset V_{\mathbf{m}} \Leftrightarrow \mathbf{n} \leqslant \mathbf{m} .
$$

Spaces $V_{\mathbf{n}}$ and $V_{\mathbf{m}}$ or grids $\Omega_{\mathbf{n}}$ and $\Omega_{\mathbf{m}}$ that satisfy this nesting relation $\mathbf{n}<\mathbf{m}$ are called related. The construction of the spaces $V_{\mathbf{n}}$ shows that even a stronger relation holds than (2), namely

$$
V_{\mathbf{n}-\mathbf{e}_{j}} \cap V_{\mathbf{n}-\mathbf{e}_{k}}=V_{\mathbf{n}-\mathbf{e}_{j}-\mathbf{e}_{k}}, \quad j, k=1,2,3, j \neq k .
$$

We also see that for $\Omega=\mathbb{R}^{3}$ the spaces $\left\{V_{\mathbf{n}}\right\}_{\mathbf{n} \in \mathbb{Z}^{3}}$ form a multiresolution analysis and that in this case the characteristic function on the unit cube, $\phi \in V_{\mathbf{o}}$,

$$
\phi(x)= \begin{cases}1 & \text { if } x \in \Omega_{\mathbf{0}, \mathbf{o}}, \\ 0 & \text { if } x \notin \Omega_{\mathbf{0}, \mathbf{0}},\end{cases}
$$

serves as the scaling function. The set $\left\{\phi_{\boldsymbol{k}}^{\mathbf{n}} \mid \phi_{\boldsymbol{k}}^{\mathbf{n}}(\boldsymbol{x})=\phi\left(2^{\mathbf{n}} \boldsymbol{x}-\boldsymbol{k}\right), \boldsymbol{k} \in \mathbb{Z}^{3}\right\}$ forms a basis in $V_{\mathrm{n}}$, which corresponds with the usual Haar-basis for the one-dimensional case.

In the case $\Omega=(0,1)^{3}$ we restrict ourselves to $V_{\mathbf{n}}$ with $n_{1}, n_{2}, n_{3} \geqslant 0$ and we see $\operatorname{dim}\left(V_{\mathbf{n}}\right)=2^{|\mathbf{n}|}$. Formally, for $\Omega=(0,1)^{3}$ and $n_{1}, n_{2}$ or $n_{3}$ negative we define $V_{\mathbf{n}}=V_{n_{1}, n_{2}, n_{3}}$ by $V_{\mathbf{n}}=\{0\}$.

${ }^{1}$ We define the inequalities between triples by and$$
\mathbf{n}<\mathbf{m} \Leftrightarrow n_{1}<m_{1}, n_{2}<m_{2}, n_{3}<m_{3},
$$$$
\mathbf{n} \leqslant \mathbf{m} \Leftrightarrow n_{1} \leqslant m_{1}, n_{2} \leqslant m_{2}, n_{3} \leqslant m_{3} \text {. }
$$ 
For all spaces $V_{\mathrm{n}}$ we introduce the restriction operator $R_{\mathrm{n}}: L^{2}(\Omega) \rightarrow V_{\mathbf{n}}$, the $L^{2}$ projection such that for $u \in L^{2}(\Omega)$ we have $R_{\mathrm{n}} u \in V_{\mathbf{n}}$ and

$$
\left(R_{\mathrm{n}} u\right)\left(z_{\mathbf{n}, k}\right)=2^{|\mathbf{n}|} \int_{\Omega_{\mathbf{n}, k}} u(z) d z
$$

\subsection{More-dimensional wavelets}

We introduce the wavelet space $W_{\mathbf{n}} \subset V_{\mathbf{n}}$ which consists of all functions in $V_{\mathbf{n}}$ that are not represented in any of the related function spaces on the next coarser level, i.e. they are in $V_{\mathbf{n}}$ but not in $\operatorname{Span}\left(V_{\mathbf{n}-\mathbf{e}_{1}}, V_{\mathbf{n}-\mathbf{e}_{2}}, V_{\mathbf{n}-\mathbf{e}_{3}}\right)$, or

$$
\begin{aligned}
V_{\mathbf{n}} & =W_{\mathbf{n}} \oplus \operatorname{Span}\left(V_{\mathbf{n}-\mathbf{e}_{1}}, V_{\mathbf{n}-\mathbf{e}_{2}}, V_{\mathbf{n}-\mathbf{e}_{3}}\right), \\
\{0\} & =W_{\mathbf{n}} \cap \operatorname{Span}\left(V_{\mathbf{n}-\mathbf{e}_{1}}, V_{\mathbf{n}-\mathbf{e}_{2}}, V_{\mathbf{n}-\mathbf{e}_{3}}\right) .
\end{aligned}
$$

This means that $W_{\mathrm{n}}$ contains the "difference information" that is available in the fine grid $V_{\mathbf{n}}$ but not in the span of the coarser grids $V_{\mathbf{n}-\mathrm{e}_{1}}, V_{\mathbf{n}-\mathrm{e}_{2}}$ and $V_{\mathbf{n}-\mathrm{e}_{3}}$.

In our case, where $V_{\mathbf{n}}$ contains the piecewise constant functions, it is simple to construct the spaces $W_{\mathbf{n}}$ such that

$$
W_{\mathbf{n}} \perp \operatorname{Span}\left(V_{\mathbf{n}-\mathbf{e}_{1}}, V_{\mathbf{n}-\mathbf{e}_{2}}, V_{\mathbf{n}-\mathbf{e}_{3}}\right) .
$$

This makes $W_{\mathbf{n}}$ the orthogonal complement of $\operatorname{Span}\left(V_{\mathbf{n}-(1,0,0)}, V_{\mathbf{n}-(0,1,0)}, V_{\mathbf{n}-(0,0,1)}\right)$ in $V_{\mathrm{n}}$, and (8) follows immediately from (9).

For $\Omega \subset \mathbb{R}^{3}$ the relation (9) allows a straightforward decomposition of $V_{\mathbf{n}}$. In the case $\Omega=\mathbb{R}^{3}$ we have ${ }^{2}$

$$
V_{\mathbf{n}}\left(\mathbb{R}^{3}\right)=\bigoplus_{j_{1}=-\infty}^{n_{1}} \bigoplus_{j_{2}=-\infty}^{n_{2}} \bigoplus_{j_{3}=-\infty}^{n_{3}} W_{j}
$$

where all $W_{j}$ are orthogonal to each other.

To handle the bounded domain $\Omega=(0,1)^{3}$ we introduce the functions $V_{\mathbf{n}}^{0}(\Omega) \subset$ $V_{\mathbf{n}}(\Omega)$ which have a zero mean value on $\Omega$, i.e. $V_{\mathbf{n}}^{0}(\Omega)=\left\{u \in V_{\mathbf{n}}(\Omega) \mid R_{\mathbf{o}}(u)=0\right\}$, and we have ${ }^{3}$

$$
V_{\mathbf{n}}^{0}(\Omega)=\bigoplus_{j_{1}=0}^{n_{1}} \bigoplus_{j_{2}=0}^{n_{2}} \bigoplus_{j_{3}=0}^{n_{3}} W_{j}
$$

and hence

$$
V_{\mathbf{n}}(\Omega)=V_{\mathbf{o}} \oplus V_{\mathbf{n}}^{0}=V_{\mathbf{o}} \oplus \bigoplus_{\mathbf{0} \leqslant j \leqslant \mathbf{n}} W_{j}
$$

${ }^{2}$ Notice that here, in the more-dimensional case, it is convenient to choose an indexing that is different from the usual indexing in the well-known one-dimensional case.

${ }^{3}$ In the case of a bounded domain, $\omega=(0,1)^{3}, W_{(0,0,0)}(\Omega)$ is the zero-function and the functions $W_{\left(j_{1}, j_{2}, j_{3}\right)}$ with some zero-index (e.g. $W_{\left(j_{1}, j_{2}, 0\right)}$ or $\left.W_{\left(0,0, j_{3}\right)}\right)$ have a shape different from those with $j>0$. 
The "difference information" between the approximations of a function $f \in L^{2}(\Omega)$ on two successive levels, $R_{\mathrm{n}} f \in V_{\mathbf{n}}$ on the one hand and $R_{\mathbf{n}-\mathbf{e}_{j}} f \in V_{\mathbf{n}-\mathbf{e}_{j}}, j=1,2,3$, on the other hand, is given by the orthogonal projection $Q_{\mathbf{n}} f$ of $f$ onto the orthogonal complement $W_{\mathbf{n}}$ of $V_{\mathbf{n}-\mathbf{e}_{j}}$ in $V_{\mathbf{n}}$. This is described in the following theorem.

\section{Theorem 2.1}

Let the operator $Q_{\mathbf{n}}: L^{2}(\Omega) \rightarrow W_{\mathbf{n}}$ be the orthogonal projection onto $W_{\mathbf{n}}$, then it follows that

$$
\begin{aligned}
Q_{\mathbf{n}} u= & R_{\mathbf{n}} u-R_{\mathbf{n}-\mathbf{e}_{1}} u-R_{\mathbf{n}-\mathbf{e}_{2}} u-R_{\mathbf{n}-\mathbf{e}_{3}} u \\
& +R_{\mathbf{n}-\mathbf{e}_{2}-\mathbf{e}_{3}} u+R_{\mathbf{n}-\mathbf{e}_{1}-\mathbf{e}_{3}} u+R_{\mathbf{n}-\mathbf{e}_{1}-\mathbf{e}_{2}} u-R_{\mathbf{n}-\mathbf{e}} u
\end{aligned}
$$

or, equivalently,

$$
Q_{\mathbf{n}} u=R_{\mathbf{n}} u-R_{\mathbf{n}-\mathbf{e}} u+\sum_{j=1,2,3}\left(R_{\mathbf{n}-\mathbf{e}+\mathbf{e}_{j}} u-R_{\mathbf{n}-\mathrm{e}_{j}} u\right) .
$$

\section{Proof}

From (10) or (12) it follows that (possibly neglecting functions in $V_{\mathbf{0}}$ if $\left.\Omega=(0,1)^{3}\right)$

$$
V_{\mathbf{n}}=\bigoplus_{j \leqslant \mathbf{n}} W_{j}
$$

so that

$$
R_{\mathrm{n}} u=\sum_{j \leqslant \mathrm{n}} Q_{j} u
$$

and

$$
\begin{aligned}
& R_{\mathbf{n}}-R_{\mathbf{n}-\mathrm{e}_{1}}-R_{\mathbf{n}-\mathrm{e}_{2}}-R_{\mathbf{n}-\mathrm{e}_{3}}+R_{\mathbf{n}-\mathrm{e}_{2}-\mathrm{e}_{3}}+R_{\mathbf{n}-\mathrm{e}_{1}-\mathrm{e}_{3}}+R_{\mathbf{n}-\mathrm{e}_{1}-\mathrm{e}_{2}}-R_{\mathbf{n}-\mathbf{e}} \\
& =\sum_{j \leqslant \mathrm{n}} Q_{j}-\sum_{j \leqslant \mathrm{n}-\mathrm{e}_{1}} Q_{j}-\sum_{j \leqslant \mathrm{n}-\mathrm{e}_{2}} Q_{j}-\sum_{j \leqslant \mathrm{n}-\mathrm{e}_{3}} Q_{j} \\
& +\sum_{j \leqslant \mathbf{n}-\mathrm{e}_{2}-\mathrm{e}_{3}} Q_{j}+\sum_{j \leqslant \mathbf{n}-\mathbf{e}_{1}-\mathrm{e}_{3}} Q_{j}+\sum_{j \leqslant \mathbf{n}-\mathbf{e}_{1}-\mathbf{e}_{2}} Q_{j}-\sum_{j \leqslant \mathbf{n}-\mathbf{e}} Q_{j} \\
& =\sum_{\mathbf{n}-\mathbf{e}<j \leqslant \mathbf{n}} Q_{j}+\sum_{\mathbf{n}-\mathbf{e}_{1}<j \leqslant \mathbf{n}} Q_{j}+\sum_{\mathbf{n}-\mathbf{e}_{2}<j \leqslant \mathbf{n}} Q_{j}+\sum_{\mathbf{n}-\mathbf{e}_{3}<j \leqslant n} Q_{j} \\
& -\sum_{n-e_{2}-e_{3}<j \leqslant n} Q_{j}-\sum_{n-e_{1}-e_{3}<j \leqslant n} Q_{j}-\sum_{n-e_{1}-e_{2}<j \leqslant n} Q_{j} \\
& =Q_{\mathrm{n}} \text {. }
\end{aligned}
$$

\section{Remarks}

- In the right-hand-side of equation (13) we recognise the information that can be represented on the levels $|\mathbf{n}|,|\mathbf{n}|-3,|\mathbf{n}|-2,|\mathbf{n}|-1$, respectively. 
- In (13) the information on the levels $|\mathbf{n}|-2$ and $|\mathbf{n}|-3$ can directly be derived from the information on level $|\mathbf{n}|-1$, e.g. by $R_{\mathbf{n}-\mathbf{e}_{2}-\mathbf{e}_{3}} u=R_{\mathbf{n}-\mathbf{e}_{2}-\mathbf{e}_{3}}\left(R_{\mathbf{n}-\mathbf{e}_{3}} u\right)$. Thus, expression (13) describes the difference information between $R_{\mathrm{n}} u$ and its approximation on the related next coarser grids.

- Notice that in the two-dimensional case the relation (13) reduces to

$$
Q_{\mathrm{n}} u=R_{\mathrm{n}} u-R_{\mathrm{n}-\mathrm{e}_{1}} u-R_{\mathrm{n}-\mathrm{e}_{2}} u+R_{\mathrm{n}-\mathrm{e}} u,
$$

where $\mathbf{e}=(1,1)$, and in the one-dimensional case we have

$$
Q_{\mathrm{n}} u=R_{\mathrm{n}} u-R_{\mathrm{n}-\mathrm{e}} u .
$$

First, in the remainder of this section we restrict ourselves to the case of the unbounded domain $\Omega=\mathbb{R}^{3}$. The four relations (1.1) to (1.4) imply that also the spaces $W_{\mathbf{n}}$ are scaled versions of one space $W_{\mathbf{0}}$,

$$
f(\boldsymbol{x}) \in W_{\mathbf{n}} \Leftrightarrow f\left(2^{-\mathbf{n}} \boldsymbol{x}\right) \in W_{\mathbf{0}}, \quad \forall \mathbf{n} \in \mathbb{Z}^{3},
$$

and, moreover, that they are translation invariant for the discrete translations $2^{-\mathbf{n}} \mathbb{Z}^{3}$

$$
f(\boldsymbol{x}) \in W_{\mathbf{0}} \Leftrightarrow f(\boldsymbol{x}-\boldsymbol{k}) \in W_{\mathbf{0}}, \quad \forall \mathbf{n} \in \mathbb{Z}^{3} .
$$

The relations (7) and (9) make that they are mutually orthogonal spaces, generating all functions of $L^{2}\left(\mathbb{R}^{3}\right)$,

$$
\begin{aligned}
& W_{\mathbf{n}} \perp W_{\mathbf{m}} \quad \text { for } \mathbf{n} \neq \mathbf{m}, \\
& \bigoplus_{\mathbf{n} \in \mathbb{Z}^{3}} W_{\mathbf{n}} \subset_{\text {dense }} L^{2}\left(\mathbb{R}^{3}\right) .
\end{aligned}
$$

Summarising, we obtain a nesting between the spaces $\left\{V_{\mathbf{n}}\right\}$ and $\left\{W_{\mathbf{n}}\right\}$,

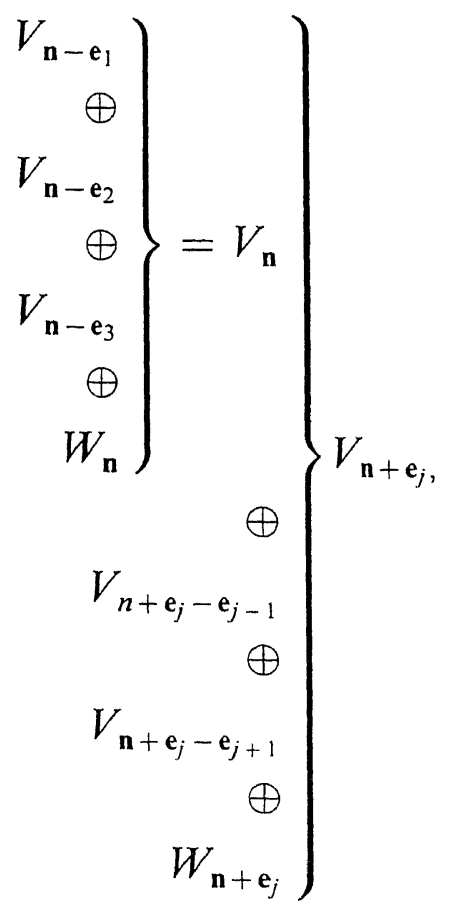


that is essentially more complex than in the classical one-dimensional case, where there is a sequential ordering of the spaces $\left\{V_{\mathbf{n}}\right\}$ and $\left\{W_{\mathbf{n}}\right\}$.

As soon as we find a function $\psi(\boldsymbol{x})$ with the property that $\psi(\boldsymbol{x}-\boldsymbol{k}), k \in \mathbb{Z}^{3}$, is a basis of $W_{\mathrm{e}}$, then by a simple rescaling, we see that $\psi\left(2^{\mathbf{n}} \boldsymbol{x}-\boldsymbol{k}\right)$ yields a basis of $W_{\mathrm{n}+\mathrm{e}}$. Such a function is the more-dimensional generalisation of a wavelet. Since $L^{2}\left(\mathbb{R}^{3}\right)$ is the direct sum of these $W_{\mathbf{n}+\mathrm{e}}$, the full collection $\left\{\psi_{k}^{\mathbf{n}+\mathbf{e}}(\boldsymbol{x}) \mid \psi_{k}^{\mathbf{n}+\mathbf{e}}(\boldsymbol{x})=\right.$ $\left.\psi\left(2^{\mathrm{n}} \boldsymbol{x}-\boldsymbol{k}\right), \mathbf{n}, \boldsymbol{k} \in \mathbb{Z}^{3}\right\}$ is a basis of $L^{2}\left(\mathbb{R}^{3}\right)$.

It is easy to check that the more-dimensional wavelet $\psi(\boldsymbol{x}) \in W_{\mathrm{e}}$, corresponding with the scaling function $\phi(\boldsymbol{x}) \in V_{0}$, from the previous section, is the three-dimensional checkerboard basis function ${ }^{4}$ given by (5):

$$
\psi(\boldsymbol{x})=\left\{\begin{array}{llll}
=0 & \text { if } \boldsymbol{x} \notin \Omega_{\mathbf{o}, \mathbf{o}}, & \\
=+1 & \text { if } \boldsymbol{x} \in \Omega_{\mathbf{o}, \mathbf{o}}, & \boldsymbol{x} \in \Omega_{\mathbf{e}, \boldsymbol{k}}, & |\boldsymbol{k}| \text { even } \\
=-1 & \text { if } \boldsymbol{x} \in \Omega_{\mathbf{o}, \mathbf{o}}, & \boldsymbol{x} \in \Omega_{\mathbf{e}, \boldsymbol{k}}, & |\boldsymbol{k}| \text { odd }
\end{array}\right.
$$

This function is the three-dimensional generalisation of the Haar-wavelet.

In wavelet theory the spaces $W_{\mathrm{n}}$ are labelled channels, and the distinct channels are linearly independent. The first decomposition of an arbitrary function from $L^{2}(\Omega)$ with $\Omega=\mathbb{R}^{3}$ consists in writing $u(\boldsymbol{x})=\sum_{\mathbf{n}} u_{\mathbf{n}}(\boldsymbol{x})$, where each $u_{\mathbf{n}}$ belongs to the corresponding channel $W_{\mathbf{n}}$ with $\mathbf{n} \in \mathbb{Z}^{3}$.

Similarly, we can write for functions defined on $\Omega=(0,1)^{3}$ the relation (12) and make a similar decomposition in channels. Each subspace $W_{\mathbf{n}+\mathbf{e}}, \mathbf{n} \geqslant \mathbf{0}$, has its natural basis, the standard basis ${ }^{5}$

$$
\left\{\psi_{\boldsymbol{k}}^{\mathbf{n}+\mathbf{e}}(\boldsymbol{x}) \mid \psi_{\boldsymbol{k}}^{\mathbf{n}+\mathbf{e}}(\boldsymbol{x})=\psi\left(2^{\mathbf{n}} \boldsymbol{x}-\boldsymbol{k}\right)\right\}
$$

of functions with a minimal support. The basis function $\psi_{\boldsymbol{k}}^{\mathbf{n}+\mathbf{e}}$ is a scaled, elementary checkerboard function, that may be characterised either by its support which is a single cell in $\Omega_{\mathrm{n}}$ or by the centerpoints of this cell, $\mathbf{z}_{\mathbf{n}, k}$.

For $\Omega=(0,1)^{3}$, the exceptions related with the boundary are found in the spaces $W_{\mathbf{n}}$ with a zero index (i.e. $n_{1} \cdot n_{2} \cdot n_{3}=0$ ). These $W_{\mathbf{n}}$ have basis functions with different shapes. They are derived from the corresponding functions for the unbounded case, but their support is restricted to $\Omega_{\mathbf{n}-\mathrm{e}} \cap \Omega$. Their corresponding nodal points $\mathbf{z}_{\mathbf{n}-\mathbf{e}, k}$ are found on the boundary $\partial \Omega=\bar{\Omega} \backslash \Omega, \mathbf{n} \leqslant \mathbf{e}, \mathbf{n} \neq \mathbf{0}$. Taking this into account, both for $\Omega=(0,1)^{3}$ and for $\Omega=\mathbb{R}$ we may write for each $u \in L^{2}(\Omega)$ a wavelet expansion

$$
u(\boldsymbol{x})=\sum_{\mathbf{n}, \boldsymbol{k}} a_{\mathbf{n}, \boldsymbol{k}} \psi\left(2^{\mathbf{n}} \boldsymbol{x}-\boldsymbol{k}\right)
$$

\footnotetext{
${ }^{4}$ Notice $\psi \in W_{\mathbf{e}} \subset V_{\mathrm{e}}$ is a function piecewise constant on $\Omega_{\mathrm{e}}$.

${ }^{5}$ Notice that in more dimensions we use the indexing $\psi_{k}^{\mathrm{n}+\mathrm{e}}$, whereas in the one-dimensional case one usually writes $\psi_{k}^{n}$.
} 


\subsection{Approximation results}

The decompositions (10) or (12) clearly allow the approximation of a sufficiently smooth function in $L^{2}(\Omega)$ by a series with elements in $W_{j}$. To obtain an impression of the quality of these expansions we derive some error estimates.

As the case where boundaries are present is the more general one, we take $\Omega=(0,1)^{3}$. To quantify the error of approximation on $\Omega$, we introduce for $u \in C^{3}(\bar{\Omega})$ the seminorm ${ }^{6}$

$$
|u|=\sup _{\boldsymbol{x} \in \Omega}\left|\frac{\partial^{3} u(\boldsymbol{x})}{\partial x_{1} \partial x_{2} \partial x_{3}}\right|+\max _{p, q, r=0,1} \sup _{\boldsymbol{x} \in \partial \Omega}\left|\left(\frac{\partial}{\partial x_{1}}\right)^{p}\left(\frac{\partial}{\partial x_{2}}\right)^{q}\left(\frac{\partial}{\partial x_{3}}\right)^{r} u(\boldsymbol{x})\right| .
$$

Now we derive the following

\section{Theorem 2.2}

If we consider an expansion of a $C^{3}(\bar{\Omega})$-function, $u$, in piecewise constant functions on the grid $\Omega_{\mathbf{n}}$, for an arbitrary $\mathbf{n} \in \mathbb{Z}^{3}, \mathbf{n}>\mathbf{o}$, and if we write

$$
R_{\mathrm{n}} u=v_{\mathrm{o}}+\sum_{\mathbf{0} \leqslant j \leqslant \mathrm{n}} u_{j}
$$

with $v_{\mathbf{0}} \in V_{\mathbf{0}}$ and $u_{j} \in W_{j}, \mathbf{o} \leqslant \mathbf{j} \leqslant \mathbf{n}$, then

$$
\left\|u_{j}\right\|_{L^{2}(\Omega)} \leqslant 2^{-3|j| / 2}|u|
$$

and we get an estimate for the approximation error

$$
\begin{aligned}
\left\|u-R_{\mathrm{n}} u\right\|_{L^{2}(\Omega)} & \leqslant 8 \cdot 7^{-3 / 2}\left(2^{-3 n_{1}}+2^{-3 n_{2}}+2^{-3 n_{3}}\right)^{1 / 2}|u| \\
& \leqslant 8 \cdot 7^{-3 / 2}\left(h_{1}^{3 / 2}+h_{2}^{3 / 2}+h_{3}^{3 / 2}\right)|u| .
\end{aligned}
$$

\section{Proof}

We take the normalised $\left\{\tilde{\psi}_{k}^{j}\right\}=\left\{2^{|j-\mathrm{e}| / 2} \psi_{k}^{j}\right\}$ as a basis in $W_{j}, \mathbf{0} \leqslant \boldsymbol{j} \leqslant \mathbf{n}, \boldsymbol{j} \neq \mathbf{o}$. Clearly, all those functions are orthogonal to all functions $v_{0} \in V_{0}$ and mutually they form an orthonormal set (an orthonormal Haar basis) in $W_{j} \subset L^{2}(\Omega)$. We see this as follows

$$
\begin{array}{ll}
\psi \in W_{\mathrm{e}}, & \text { support }(\psi)=\Omega_{\mathbf{o}, \mathbf{e}}, \\
\psi_{k}^{\mathrm{e}} \in W_{\mathrm{e}}, & \operatorname{support}\left(\psi_{\boldsymbol{k}}^{\mathrm{e}}\right)=\Omega_{\mathbf{o}, \boldsymbol{k}}, \\
\psi_{\boldsymbol{k}}^{j} \in W_{\mathrm{e}}, & \operatorname{support}\left(\psi_{\boldsymbol{k}}^{j}\right)=\Omega_{\boldsymbol{j}-\mathrm{e}, \boldsymbol{k}},
\end{array}
$$

or, in other words, $\psi_{\boldsymbol{k}}^{j} \in V_{\boldsymbol{j}}$, but $\psi_{\boldsymbol{k}}^{j}$ scales like a basis function in $V_{\boldsymbol{j}-\mathrm{e}}$. Hence

$$
\int 2^{|j-\mathbf{e}| / 2} \psi_{k}^{j} 2^{|j-\mathbf{e}| / 2} \psi_{\mathbf{m}}^{j} d \Omega=0 \quad \text { for } \boldsymbol{k} \neq \mathbf{m}
$$

\footnotetext{
${ }^{6}$ The necessity of the boundary terms in this seminorm is seen immediately if we want to approximate in $L^{2}(\Omega)$ smooth functions $u \in C^{3}(\bar{\Omega})$ that do not satisfy homogeneous boundary conditions.
} 
and

$$
\int 2^{|j-\mathbf{e}| / 2} \psi_{k}^{j} 2^{|j-\mathbf{e}| / 2} \psi_{k}^{j} d \Omega=2^{|j-\mathbf{e}|} \int \psi_{k}^{j} \psi_{k}^{j} d \Omega=2^{|j-\mathbf{e}|} \int_{\Omega_{j-\mathbf{e} . k}} d \Omega=1 .
$$

Thus, we find

with

$$
R_{\mathrm{n}} u=v_{0}+\sum_{0 \leqslant j \leqslant \mathrm{n}} u_{j}
$$

Now

$$
u_{j}=\sum_{k} a_{j k} \tilde{\psi}_{k}^{j}=\sum_{k}\left(u, \tilde{\psi}_{k}^{j}\right) \tilde{\psi}_{k}^{j}
$$

$$
a_{j k}=\left(u, \tilde{\psi}_{k}^{j}\right)=\int_{\Omega} u \tilde{\psi}_{k}^{j} d \Omega=\int_{\Omega_{j-\mathrm{e}, k}} u \tilde{\psi}_{k}^{j} d \Omega
$$

By Taylor expansion around $\mathbf{z}_{\mathbf{j}-\mathbf{e}, \boldsymbol{k}}$, we have

$$
\left|\int_{\Omega_{j-\mathbf{e}, k}} u \tilde{\psi}_{k}^{j} d \Omega\right| \leqslant 2^{-2|j|}|u| .
$$

For $\boldsymbol{j} \geqslant \mathbf{e}$ the point $\mathbf{z}_{\boldsymbol{j}-\mathbf{e}, \boldsymbol{k}}$ lies in the interior of $\Omega$ and the estimate holds with

$$
|u|=\sup _{x \in \Omega}\left|\frac{\partial^{3} u(\boldsymbol{x})}{\partial x_{1} \partial x_{2} \partial x_{3}}\right|
$$

For $\boldsymbol{j} \not \mathrm{e}$, i.e. for $\psi_{\boldsymbol{k}}^{j}$ with $\boldsymbol{j}$-component equal to zero, the point $\mathbf{z}_{\boldsymbol{j}-\mathrm{e}, \boldsymbol{k}}$ lies on the boundary and the function $\psi_{k}^{j}$ is constant in one direction over the whole domain $\Omega$, and it is of Haar-wavelet type for the non-zero indices (or index). In this situation the same estimate (24) holds with, e.g. if $j_{1}=0$,

$$
|u|=\sup \left|\frac{\partial^{2} u(\boldsymbol{x})}{\partial x_{2} \partial x_{3}}\right|
$$

For $\boldsymbol{j}=\mathbf{0}$ the relation (24) is trivially satisfied. Hence, the estimate (24) holds for $j \geqslant \mathbf{o}$ if we use the serminorm (21), and we find

$$
\begin{gathered}
\left|a_{j, k}\right| \leqslant 2^{-2|j|}|u| \\
\left\|u_{j}\right\|^{2}=\sum_{k}\left|a_{j k}\right|^{2} \leqslant \sum_{k} 2^{-4|j|}|u|^{2}=2^{-3|j|}|u|^{2}
\end{gathered}
$$

so that

$$
\left\|u_{j}\right\| \leqslant 2^{-3|j| / 2}|u|
$$

and

$$
\left\|u-R_{\mathrm{n}} u\right\|^{2}=\sum_{\substack{j_{1}>n_{1} \\ \text { or } j_{2}>n_{2} \\ \text { or } j_{3}>n_{3}}}\left\|u_{j}\right\|^{2} \leqslant|u|^{2}\left(\sum_{\substack{j_{1}>n_{1} \\ j_{2} \geqslant 0 \\ j_{3} \geqslant 0}}+\sum_{\substack{j_{1} \geqslant 0 \\ j_{2}>n_{2} \\ j_{3} \geqslant 0}}+\sum_{\substack{j_{1} \geqslant 0 \\ j_{2} \geqslant 0 \\ j_{3}>n_{3}}}\right) 2^{-3|j|}
$$




$$
\leqslant\left(\frac{8}{7}\right)^{3}\left(2^{-3 n_{1}}+2^{-3 n_{2}}+2^{-3 n_{3}}\right)|u|^{2} .
$$

and it follows that

$$
\begin{aligned}
\left\|u-R_{\mathrm{n}} u\right\| & \leqslant\left(\frac{8}{7}\right)^{3 / 2}\left(2^{-3 n_{1} / 2}+2^{-3 n_{2} / 2}+2^{-3 n_{3} / 2}\right)|u| \\
& =\left(\frac{8}{7}\right)^{3 / 2}\left(h_{1}^{3 / 2}+h_{2}^{3 / 2}+h_{2}^{3 / 2}\right)|u| .
\end{aligned}
$$

If we have no further a-priori knowledge about $u$, the most efficient approximation will be one with $h_{1}=h_{2}=h_{3}$ because this equalises the three main terms in the error bound. We see that

$$
R_{\mathbf{n}}=\sum_{j \leqslant \mathbf{n}} Q_{j}
$$

and the truncation error for $u-R_{\mathbf{n}} u$ is neither particularly promising nor surprising: the major part of the error is produced by the largest meshwidth: $\left(\max \left(h_{1}, h_{2}, h_{3}\right)\right)^{3 / 2}$, whereas the total number of degrees of freedom for an element in $V_{\mathbf{n}}$ is $2^{|\mathbf{n}|}$.

Following the idea of sparse grids, as introduced for finite elements in [3,9], a better accuracy per degree of freedom is obtained for the approximation operator

$$
\hat{R}_{m}=\sum_{|j| \leqslant m} Q_{j}
$$

with $m \in \mathbb{Z}$.

\section{Theorem 2.3}

For the approximation operator (25) we have the truncation error estimate

$$
\left\|u-\hat{R}_{m} u\right\|<M(\epsilon) 2^{-(3-\epsilon) m / 2}|u|=M(\epsilon)\left(h_{1} h_{2} h_{3}\right)^{(3-\epsilon) / 2}|u| .
$$

for some arbitrary small constant $\epsilon$ and a constant $M(\epsilon)$, depending on $\epsilon$.

Proof

Following the same lines as in theorem 2.2 , and because $\left.\left\|u_{j}\right\|\right|^{2} \leqslant 2^{-3|j|}|u|$, we get

$$
\begin{aligned}
\left\|u-\hat{R}_{m} u\right\|^{2} & =\sum_{|j|>m}\left\|u_{j}\right\|^{2} \\
& \leqslant|u|^{2} \sum_{|j|>m} 2^{-3|j|}=|u|^{2} \sum_{l>m|j|=l} \sum_{|j|} 2^{-3|j|} \\
& =|u|^{2} \sum_{l>m} \frac{(l+1)(1+2)}{2} 2^{-\epsilon l} 2^{-(3-\epsilon) l}
\end{aligned}
$$




$$
\begin{gathered}
\leqslant|u|^{2} M(\epsilon)^{2} \sum_{l>m} 2^{-(3-\epsilon) l} \\
=|u|^{2} M(\epsilon)^{2} 2^{-m(3-\epsilon)} . \\
\left\|u-\hat{R}_{m} u\right\| \leqslant M(\epsilon) 2^{-m(3-\epsilon) / 2}|u|,
\end{gathered}
$$

$2^{-m}=h_{1} h_{2} h_{3}$ is the volume of the smallest cells in the sparse grid used for the rimation of $u$.

22), all $h_{j}$ need to be small and in (26) only their product. This means that avergence in (22) all meshsizes should tend to zero, whereas in (26) only a should vanish. Further, the estimate (26) is of the same order of accuracy , except for a logarithmic small factor. However, the number of degrees of $\mathbf{n}$ for the approximation (26) is significantly less. Namely, in the unit For $R_{\mathbf{n}} u$ the number of degrees of freedom is $2^{|\mathbf{n}|}$, whereas for $\hat{R}_{m} u$ it is $m+2) 2^{m}-1$. Because significantly fewer degrees of freedom are involved ıpproximation $\hat{R}_{m} u$ than in the approximation of $R_{(m, m, m)} u$, i.e. fewer coeffi$\boldsymbol{\imath}_{\boldsymbol{j}, \boldsymbol{k}}$ and fewer gridpoints $\mathbf{z}_{\boldsymbol{j}, \boldsymbol{k}}$, following [3], we call the approximation $\hat{R}_{m} u$ the grid approximation and

$$
\Omega_{m}^{*}=\left\{\mathbf{z}_{\boldsymbol{j}, \boldsymbol{k}}\left|\mathbf{z}_{\boldsymbol{j}, \boldsymbol{k}} \in \Omega_{\boldsymbol{j}}^{*},\right| \boldsymbol{j} \mid \leqslant m\right\}
$$

parse grid or sparse box grid for this approximation on level $\mathrm{m}$.

is paper we are interested in the approximate solution of PDEs discretised larse grid, i.e. we are looking for an approximation of the solution of these ,ns in the space

$$
S_{m}\left(\mathbb{R}^{3}\right)=\bigoplus_{|\mathbf{n}| \leqslant m} V_{\mathbf{n}}=\bigoplus_{|\mathbf{n}| \leqslant m} W_{\mathbf{n}}
$$

$\Omega=(0,1)^{3}$, in the space

$$
S_{m}(\Omega)=\bigoplus_{0 \leqslant|\mathbf{n}| \leqslant m} V_{\mathbf{n}}=V_{\mathbf{0}} \oplus \bigoplus_{\mathbf{0} \leqslant|\mathbf{n}| \leqslant m} W_{\mathbf{n}} .
$$

$S_{m}(\Omega)$ the mth level sparse-grid space.

\section{multigrid algorithm}

basis principle of multigrid for the solution of the discrete equation

$$
L_{h} u_{h}=f_{h}
$$

the high frequencies in the error are reduced by relaxation on a fine grid, s the low frequencies are taken care of by coarse-grid discrete equations. 
The classical coarse grid correction (CGC) step is described by

$$
u^{\text {(new) }}=u^{\text {(old) }}+P_{h H} L_{H}^{-1} R_{H h}\left(f_{h}-L_{h} u^{\text {(old })}\right)
$$

where $L_{H}$ is the coarse-grid discrete operator and $P_{h H}$ and $R_{H h}$ are the grid-transfer operators from the coarse-to-the-fine and fine-to-the-coarse grid respectively. Uusually the coarse-grid mesh size is twice the mesh size on the next finer grid. The coarse grid problem is approximately solved by means of the recursive application of the same algorithm on the coarser level. In this classical procedure a linearly ordered sequence of fine and coarse discretisations is required.

In the case of our sparse-grid finite-volume approximation, a discretisation should exist for all grids $\Omega_{\mathbf{n}},|\mathbf{n}| \leqslant m$, fine and coarse. On each of these grids we can obtain approximations to $u_{\mathrm{n}} \in V_{\mathrm{n}}$, the solution of the discrete problem

$$
L_{\mathbf{n}} u_{\mathbf{n}}=f_{\mathbf{n}} \text { on } \Omega_{\mathbf{n}} \text {. }
$$

These discretisations, however, do not offer an ordered sequence. Nevertheless, the multidimensional wavelet decomposition of $u_{\mathbf{n}} \in V_{\mathbf{n}}$,

$$
u_{\mathrm{n}}=v_{\mathbf{0}}+\sum_{j \leqslant \mathrm{n}} w_{j}, \quad \text { with } w_{j} \in W_{j},
$$

allows us to distinguish a high-frequency component, $w_{\mathrm{n}}$, that cannot be represented on coarser grids, and all other components, $v_{\mathbf{o}}$ and $w_{\boldsymbol{j}}, \boldsymbol{j} \leqslant \mathbf{n}, \boldsymbol{j} \neq \mathbf{n}$, which can be present in coarser grid representations. Therefore we may consider the grid $\Omega_{\mathbf{n}}$ to be solely responsible for the accurate (and efficient) representation of $w_{\mathbf{n}}$. This component is clearly a high-frequency function (in fact a checkerboardtype function), of which an error can be efficiently reduced by a simple relaxation procedure as e.g. damped Jacobi.

The decomposition (13) in theorem 2.1 shows us how a CGC can be obtained from these coarser grids in $\Omega_{\mathbf{n}-\mathbf{e}_{j}}, j=1,2,3$,

$$
\begin{aligned}
u_{\mathbf{n}}^{(\text {new })}=u_{\mathbf{n}}^{(\text {old })} & +\sum_{j=1,2,3} P_{\mathbf{n}, \mathbf{n}-\mathbf{e}_{j}} L_{\mathbf{n}-\mathbf{e}_{j}}^{-1} R_{\mathbf{n}-\mathbf{e}_{j}, \mathbf{n}} r_{\mathbf{n}} \\
& -\sum_{j=1,2,3} P_{\mathbf{n}, \mathbf{n}-\mathbf{e}+\mathbf{e}_{j}} L_{\mathbf{n}-\mathbf{e}+\mathbf{e}_{j}}^{-1} R_{\mathbf{n}-\mathbf{e}+\mathbf{e}_{j}, \mathbf{n}} r_{\mathbf{n}} \\
& +P_{\mathbf{n}, \mathbf{n}-\mathbf{e}} L_{\mathbf{n}-\mathbf{e}}^{-1} R_{\mathbf{n}-\mathbf{e}, \mathbf{n}} r_{\mathbf{n}},
\end{aligned}
$$

with

$$
r_{\mathbf{n}}=f_{\mathbf{n}}-L_{\mathbf{n}} u_{\mathbf{n}}^{\text {(old })}
$$

Here we denote by $R_{\mathrm{m}, \mathbf{n}}: V_{\mathbf{n}} \rightarrow V_{\mathbf{m}}, \mathbf{m} \leqslant \mathbf{n}$, the restriction operator defined by $R_{\mathrm{m}, \mathrm{n}} u_{\mathrm{n}}=R_{\mathrm{m}} u_{\mathrm{n}}$ for all $u_{\mathrm{n}} \in V_{\mathrm{n}} \subset L^{2}(\Omega)$. The prolongation operator $P_{\mathrm{n}, \mathrm{m}}: V_{\mathrm{m}} \rightarrow$ $V_{\mathrm{n}}$ can be defined e.g. as the adjoint of $R_{\mathrm{m}, \mathrm{n}}$.

The third remark following theorem 2.1 shows how the two- or one-dimensional case can be treated similarly and we see that - for the one-dimensional case - our approach reduces to the classical scheme. 
The approach (30) would imply three coarser levels to be active for a CGC, and - as was shown in the remark after theorem 2.1 - we can do with only one coarser level by deriving the information on the levels $|\mathbf{n}|-3$ and $|\mathbf{n}|-2$ from the information on level $|\mathbf{n}|-1$. If we consider the corrections from level $|\mathbf{n}|-1$,

$$
c_{\mathbf{n}-\mathbf{e}_{j}}=L_{\mathbf{n}-\mathbf{e}_{j}}^{-1} R_{\mathbf{n}-\mathbf{e}_{j}} R_{\mathbf{n}-\mathbf{e}_{j}, \mathbf{n}} r_{\mathbf{n}}, \quad j=1,2,3,
$$

as approximating a single (but unknown) correction function $c_{\mathrm{n}} \in V_{\mathrm{n}}$, the corrections from the levels $|\mathbf{n}|-2$ and $|\mathbf{n}|-3$ can be computed as the mean values

$$
c_{\mathbf{n}-\mathbf{e}+\mathbf{e}_{j}}=\frac{1}{2}\left(R_{\mathbf{n}-\mathbf{e}+\mathbf{e}_{j}, \mathbf{n}-\mathbf{e}_{j+1}} c_{\mathbf{n}-\mathbf{e}_{j+1}}+R_{\mathbf{n}-\mathbf{e}+\mathbf{e}_{j}, \mathbf{n}-\mathbf{e}_{j-1}} c_{\mathbf{n}-\mathbf{e}_{j-1}}\right),
$$

$j=1,2,3$, and

$$
c_{\mathbf{n}-\mathbf{e}}=\frac{1}{3} \sum_{j=1,2,3} R_{\mathbf{n}-\mathbf{e}, \mathbf{n}-\mathbf{e}+\mathbf{e}_{j}} c_{\mathbf{n}-\mathbf{e}+\mathbf{e}_{j}} .
$$

This is justified by the fact that the restrictions are commutative, i.e.

$$
\mathbf{m} \leqslant \mathbf{n} \leqslant l \Rightarrow R_{\mathbf{m}, \mathbf{n}} R_{\mathrm{n}, l}=R_{\mathrm{m}, l}
$$

and the following (trivial) lemma.

\section{Lemma 3.1}

If all discrete operators $L_{\mathbf{n}}$ are stable and relatively consistent, i.e.

$$
\left\|R_{\mathbf{n}, \mathbf{n}+\mathbf{e}_{j}} L_{\mathbf{n}+\mathbf{e}_{j}}-L_{\mathbf{n}} R_{\mathbf{n}, \mathbf{n}+\mathrm{e}_{j}}\right\| \leqslant \mathcal{O}\left(2^{-|\mathbf{n}| p}\right),
$$

then

$$
\left\|c_{\mathbf{n}-\mathbf{e}_{j}}-R_{\mathbf{n}-\mathbf{e}_{j}, \mathbf{n}} c_{\mathbf{n}}\right\| \leqslant \mathcal{O}\left(2^{-|\mathbf{n}| p}\right)
$$

The consistent discretisations can be derived e.g. from the fine grid discretisation $L_{\mathbf{n}}$ by taking the Galerkin approximation

$$
L_{\mathbf{n}-\mathbf{e}_{j}}=R_{\mathbf{n}-\mathbf{e}_{j}, \mathbf{n}} L_{\mathbf{n}} P_{\mathbf{n}, \mathbf{n}-\mathbf{e}_{j}} .
$$

If the three corrections $c_{\mathbf{n}-\mathbf{e}_{j}}$ were all restrictions of the (unknown) correction $c_{\mathbf{n}} \in V_{\mathbf{n}}$ indeed, then $R_{\mathbf{n}-\mathbf{e}+\mathbf{e}_{j}, \mathbf{n}-\mathbf{e}_{j+1}} c_{\mathbf{n}-\mathbf{e}_{j+1}}$ and $R_{\mathbf{n}-\mathbf{e}+\mathbf{e}_{j}, \mathbf{n}-\mathbf{e}_{j-1}} c_{\mathbf{n}-\mathbf{e}_{j-1}}$ would both have delivered the same result, viz., $R_{\mathbf{n}-\mathbf{e}+\mathbf{e}_{j}, \mathbf{n}} c_{\mathrm{n}}$. This gives the possibility to check how well such a function $c_{\mathrm{n}}$ can be determined, by monitoring the quantities, $j=1,2,3$,

$$
d_{\mathbf{n}-\mathbf{e}+\mathbf{e}_{j}}=\frac{1}{2}\left(R_{\mathbf{n}-\mathbf{e}+\mathbf{e}_{j}, \mathbf{n}-\mathbf{e}_{j+1}} c_{\mathbf{n}-\mathbf{e}_{j+1}}-R_{\mathbf{n}-\mathbf{e}+\mathbf{e}_{j}, \mathbf{n}-\mathbf{e}_{j-1}} c_{\mathbf{n}-\mathbf{e}_{j-1}}\right) .
$$

Summarising, our multigrid algorithm now reads:

$$
\begin{aligned}
u_{\mathbf{n}}^{(i+1)}=u_{\mathbf{n}}^{(i)} & +\sum_{j=1,2,3} P_{\mathbf{n}, \mathbf{n}-\mathbf{e}_{j}} c_{\mathbf{n}-\mathbf{e}_{j}} \\
& -\sum_{j=1,2,3} P_{\mathbf{n}, \mathbf{n}-\mathbf{e}+\mathbf{e}_{j}} c_{\mathbf{n}-\mathbf{e}+\mathbf{e}_{j}} \\
& +P_{\mathbf{n}, \mathbf{n}-\mathbf{e}} c_{\mathbf{n}, \mathbf{n}-\mathbf{e}}
\end{aligned}
$$


where the corrections are given by (32), (33) and (34). This appears to be much similar to a multigrid algorithm by semi-coarsening, proposed by Mulder in [7]. The main difference being that Mulder computes an approximation on the full grid $R_{\mathrm{n}}$, whereas we compute the sparse grid approximation $\hat{R}_{m}$.

The result of our algorithm is a solution on a sparse grid, i.e. a set of approximate solutions, viz. $\left\{u_{\mathbf{n}}|| \mathbf{n} \mid=m\right\}$, that are the solutions of the discrete equations $L_{\mathrm{n}} u_{\mathrm{n}}=f_{\mathrm{n}}$. All approximations $u_{\mathrm{n}}$ representing the same solution $u$ of the continuous problem, we assume that they approximate the $L^{2}(\Omega)$-projection of $u$ in $V_{\mathbf{m}}=V_{(m, m, m)}$. To approximate this $R_{\mathrm{m}} u \in V_{\mathrm{m}}$, we can construct a unique function $u_{\mathbf{m}} \in V_{\mathbf{m}}$ by means of the recursive interpolation formula that immediately follows from theorem 2.1:

$$
u_{k}=\sum_{j=1,2,3}\left(u_{k-\mathbf{e}_{j}}-u_{k-\mathbf{e}+\mathbf{e}_{j}}\right)+u_{k-\mathbf{e}}
$$

where $\mathbf{o} \leqslant \boldsymbol{k} \leqslant \mathbf{m},|\boldsymbol{k}|=m+1, \ldots, 3 m ; u_{\boldsymbol{k - e _ { j }}}$ are the functions computed in the previous recursion cycle and $u_{k-\mathbf{e}+\mathbf{e}_{j}}$ and $u_{k-\mathbf{e}}$ are approximations (possibly) derived as (33) and (34). In this way we finally obtain the unique representation

$$
u_{\mathbf{m}}=v_{\mathbf{o}}+\sum_{0 \leqslant|k| \leqslant m} w_{k}
$$

or

$$
u_{\mathrm{m}} \in S_{m}(\Omega) \subset V_{(m, m, m)}(\Omega) .
$$

This representation can be considered as the computed solution.

The same construction can be realised by a "decomposition and reconstruction" algorithm as used in wavelet theory [2]. Then the available approximate solutions $\left\{u_{\mathbf{n}}\right\}$ are decomposed into their components $v_{\mathbf{o}}$ and $\left\{w_{k}\right\}$ by

$$
v_{\mathbf{0}}=\frac{\sum_{|\mathbf{n}|=m} R_{\mathbf{0}} u_{\mathbf{n}}}{\sum_{|\mathbf{n}|=m} 1} \text { and } w_{\boldsymbol{k}}=\frac{\sum_{|\mathbf{n}|=m} Q_{k} u_{\mathbf{n}}}{\sum_{|\mathbf{n}|=m} 1}
$$

and the reconstruction is performed by (38). This can conveniently be performed by a kind of a "pyramid algorithm". This will be reported in a later paper.

In practice, by the choice of the discrete operator our assumption that the $L^{2}(\Omega)$ projection of $u$ was indeed consistently approximated on $V_{\mathrm{n}}$, may not necessarily hold, and it can be checked by a monitor as (35). Now, e.g. the corresponding erroneous components might be removed from the sum (38).

In the light of the treatment in section 2 it is clear what restrictions and prolongations can be used between the different grids in the multigrid process. Because $V_{\mathbf{n}} \subset L^{2}(\Omega)$, the obvious restriction $R_{\mathbf{n}-\mathbf{e}_{j}, \mathbf{n}}$ is the $L^{2}(\Omega)$-projection onto $V_{\mathbf{n}-\mathbf{e}_{j}}$ :

$$
R_{\mathrm{n}-\mathrm{e}_{j}, \mathrm{n}}=R_{\mathbf{n}-\mathbf{e}_{j}} .
$$


This makes the diagram for the restrictions commutative: for any $\boldsymbol{l} \leqslant \mathbf{m} \leqslant \mathbf{n}$ we have $R_{l, \mathrm{~m}} R_{\mathrm{m}, \mathrm{n}}=R_{l, \mathrm{n}}$.

An obvious prolongation can be the transposed restriction

$$
P_{\mathbf{n}, \mathbf{n}-\mathbf{e}_{j}}=R_{\mathbf{n}-\mathbf{e}_{j}, \mathbf{n}}^{T} \text {. }
$$

However, this prolongation being of low order, it may be more appropriate to consider higher order prolongations. Such prolongations can always be represented by an additional operator $B_{\mathbf{n}}: V_{\mathbf{n}} \rightarrow V_{\mathbf{n}}$ so that we have

$$
P_{\mathbf{n}, \mathbf{n}-\mathbf{e}_{j}}=B_{\mathbf{n}} R_{\mathbf{n}-\mathbf{e}_{j}}^{T} .
$$

Here we will not elaborate on the different possibilities for $B_{\mathrm{n}}$.

The algorithm (36) shows that all relaxation processes for $u_{\mathrm{n}}$ on one and the same level $m=|\mathbf{n}|$ can be made in parallel. The cycling between the different (scalar) levels can be made as for the classical multigrid method: we can distinguish between V-, W- or F-cycles. However, in order to prove that the convergence of our multigrid-method is independent of the meshwidth, we now have to take into account that all aspect ratios will appear in the discretisations used.

\section{Fourier convergence analysis}

In this section we first summarise some results of Fourier analysis for moredimensional discrete approximations and then we apply this to compute the convergence rate of our sparse-grid multiple-grid methods for the solution of the anisotropic Poisson equation. The approach is different from the usual treatment of Fourier analysis for multigrid for finite difference methods for the following reasons. First, in view of the discretisation of conservation laws and divergence problems, we study nested box grids. This implies that mesh points in the coarse grids do not appear in the fine grids as well. The nesting of the (box) grids is different from the usual nesting of the (finite difference or finite element) grids. Second, we do not consider the usual sequences of fine and coarser meshes for multigrid methods, but we allow $d$-dimensional $(d=2,3)$ sparse grids.

Fourier analysis is one of the common tools to analyse linear constant coefficient problems on regular grids, and it is particularly useful if the treatment of boundary conditions can be neglected.

In section 4.1 we describe general tools that can be used for the Fourier analysis of functions defined on more-dimensional box grids. The definitions and theorems provide a useful machinery for the application of local mode analysis for the multigrid box-methods. For the technical proof and details related to this section we refer to [4].

In section 4.2 we apply tools to analyse the multigrid algorithm introduced in section 3. The technical preparations in section 4.1 allow us to be brief and clear in this treatment. 


\subsection{Fourier analysis for sparse box grids}

For $u \in L^{2}\left(\mathbb{R}^{3}\right)$ we introduce its Fourier Transform (FT) $\hat{u}$, scaled as

$$
\hat{u}(\omega)=(2 \pi)^{3 / 2} \int_{\mathbb{R}^{3}} e^{-i x \omega} u(\boldsymbol{x}) d \boldsymbol{x} .
$$

Then we know that $\hat{u} \in L^{2}\left(\mathbb{R}^{3}\right)$, and a back-transformation formula is available,

$$
\breve{u}(\boldsymbol{x})=(2 \pi)^{-3 / 2} \int_{\mathbb{R}^{3}} e^{+i x \boldsymbol{x}} \hat{u}(\boldsymbol{\omega}) \mathrm{d} \boldsymbol{\omega},
$$

such that $\breve{u}(\boldsymbol{x})=u(\boldsymbol{x})$ almost everywhere on $\mathbb{R}^{3}$. Moreover, $\hat{u} \in L^{2}\left(\mathbb{R}^{3}\right)$ and Parseval's equality holds: $\|u\|_{L^{2}\left(\mathbb{R}^{2}\right)}=\|\hat{u}\|_{L^{2}\left(\mathbb{R}^{3}\right)}$.

We are interested in the Fourier transformation for an infinite set of equally spaced data. In this case the FT of such a "grid function" is a periodic function (a function defined on a torus). Therefore we introduce a few definitions.

Let $\boldsymbol{h} \in \mathbb{R}^{3}, \boldsymbol{h}>\mathbf{0}$, be given, then the $\boldsymbol{h}$-periodisation of a function $u: \mathbb{R}^{3} \rightarrow \mathbb{C}$ is defined by

$$
\tilde{u}(\boldsymbol{x})=\sum_{\boldsymbol{k} \in \mathbb{Z}^{3}} u(\boldsymbol{x}-\boldsymbol{k} \boldsymbol{h})
$$

where $\boldsymbol{k} \boldsymbol{h}=\left(k_{1} h_{1}, k_{2} h_{2}, k_{3} h_{3}\right)$. We also introduce a notation for the threedimensional torus

$$
T_{\boldsymbol{h}}=(-\pi / \boldsymbol{h}, \pi / \boldsymbol{h}]=\left(-\pi / h_{1}, \pi / h_{1}\right] \times \ldots \times\left(-\pi / h_{3}, \pi / h_{3}\right] .
$$

Further, we need the functions $\Pi$ and Sinc $\left[1\right.$, pp. 62,67] on $\mathbb{R}^{3}$,

$$
\Pi(x)= \begin{cases}1 & \text { for }\left|x_{i}\right|<1 / 2, \quad 1 \leqslant i \leqslant 3, \\ 0 & \text { otherwise }\end{cases}
$$

and

$$
\operatorname{Sinc} x=\prod_{i=1}^{i=3} \frac{\sin \pi x_{i}}{\pi x_{i}}
$$

Using the relations mentioned in $[1$, p. 98] we find

$$
\hat{\Pi}(\boldsymbol{\omega})=(2 \pi)^{-3 / 2} \prod_{i=1,2,3} \operatorname{sinc}\left(\frac{\omega_{i}}{2 \pi}\right)=(2 \pi)^{-3 / 2} \operatorname{Sinc}\left(\frac{\omega}{2 \pi}\right) .
$$

For an $\boldsymbol{h} \in \mathbb{R}^{3}, \boldsymbol{h}>\mathbf{o}$, we define the dilation operator $D_{h}: L^{2}\left(\mathbb{R}^{3}\right) \rightarrow L^{2}\left(\mathbb{R}^{3}\right)$ by

$$
D_{\boldsymbol{h}} f(\boldsymbol{x})=h^{-3 / 2} f(\boldsymbol{x} \boldsymbol{h}),
$$

where $h=\left(h_{1} h_{2} h_{3}\right)^{1 / 3}$, and the convolution operator, $\star$, by

$$
(f \star g)(\boldsymbol{x})=(2 \pi)^{-3 / 2} \int_{\mathbb{R}^{3}} f(\boldsymbol{y}) g(\boldsymbol{x}-\boldsymbol{y}) \mathrm{d} \boldsymbol{y} .
$$


We now know that

$$
\widehat{D_{\boldsymbol{h}}}=D_{1 / \boldsymbol{h}} \hat{f} \quad \text { and } \quad \widehat{f \star g}(\boldsymbol{\omega})=\hat{f}(\boldsymbol{\omega}) \cdot \hat{g}(\boldsymbol{\omega})
$$

\subsubsection{Grid functions}

Here we introduce notations for the different types of grids and grid functions.

\section{Definition 4.1}

For a fixed mesh parameter $\boldsymbol{h} \in \mathbb{R}^{3}, \boldsymbol{h}>\mathbf{o}$, and for $\boldsymbol{j} \in \mathbb{Z}^{3}$, we define an elementary cell by $\Omega_{\boldsymbol{h}, \boldsymbol{j}}=\{\boldsymbol{x} \mid \boldsymbol{j} \boldsymbol{h}<\boldsymbol{x}<(\boldsymbol{j}+\mathbf{e}) \boldsymbol{h}\}$, the volume of the cell is denoted by $h^{3}=h_{1} \cdot h_{2} \cdot h_{3}$, and the box-grid is defined by $\Omega_{\boldsymbol{h}}=\left\{\Omega_{\boldsymbol{h}, \boldsymbol{j}} \mid \boldsymbol{j} \in \mathbb{Z}^{3}\right\}$. The regular infinite three-dimensional grid of vertices $\mathbb{Z}_{\boldsymbol{h}}$ is defined by $\mathbb{Z}_{\boldsymbol{h}}=\left\{\boldsymbol{j} \boldsymbol{h} \mid \boldsymbol{j} \in \mathbb{Z}^{3}\right\}$, which should be well distinguished from the shifted grid which is defined by $\mathbb{Z}_{\boldsymbol{h}}^{\star}=\left\{(\boldsymbol{j}+\mathrm{e} / 2) \boldsymbol{h} \mid \boldsymbol{j} \in \mathbb{Z}^{3}\right\}$.

Notice the relation with the grids as defined in section 2.2: $\Omega_{\mathbf{n}}$ can be considered as a special case of $\Omega_{h}$, and $\Omega_{h}^{\star}$ as a special case of $\mathbb{Z}_{\boldsymbol{h}}^{\star}$.

\section{Definition 4.2}

A complex or a real grid function $u_{\boldsymbol{h}}^{\bullet}$ is a mapping $\mathbb{Z}_{\boldsymbol{h}} \rightarrow \mathbb{C}$, or $\mathbb{Z}_{\boldsymbol{h}} \rightarrow \mathbb{R}$, and a shifted or box-grid function $u_{h}^{\star}$ is a mapping $\mathbb{Z}_{h}^{\star} \rightarrow \mathbb{C}$ or $\mathbb{Z}_{h}^{\star} \rightarrow \mathbb{R}$.

The vector space of such grid functions we denote by $l\left(\mathbb{Z}_{\boldsymbol{h}}\right)$ or $l\left(\mathbb{Z}_{\boldsymbol{h}}^{\star}\right)$, or briefly, by l. For any $p \geqslant 1$ the space $l\left(\mathbb{Z}_{\boldsymbol{h}}\right)$ can be provided with a norm $\|\cdot\|_{p}$

$$
\left\|u_{\boldsymbol{h}}^{\bullet}\right\|_{p}=\left(h^{3} \sum_{\boldsymbol{j} \in \mathbb{Z}^{3}}\left|u_{\boldsymbol{h}}^{\bullet}(\boldsymbol{j} \boldsymbol{h})\right|^{p}\right)^{1 / p} .
$$

For a fixed $p$, all grid functions with a finite norm $\|\cdot\|_{p}$ form a Banach space denoted by $l^{p}\left(\mathbb{Z}_{\boldsymbol{h}}\right)$. For $p=2$ we know that $l^{2}\left(\mathbb{Z}_{\boldsymbol{h}}\right)$ is a Hilbert space with the inner product

$$
\left\langle u_{\boldsymbol{h}}^{\bullet}, v_{\boldsymbol{h}}^{\bullet}\right\rangle_{l^{2}\left(\mathbb{Z}_{\boldsymbol{h}}\right)}=h^{3} \sum_{\boldsymbol{j} \in \mathbb{Z}^{3}} u_{\boldsymbol{h}}^{\bullet}(\boldsymbol{j} \boldsymbol{h}) \overline{v_{\boldsymbol{h}}^{\bullet}}(\boldsymbol{j} \boldsymbol{h}) \quad \text { with } u_{\boldsymbol{h}}^{\bullet}, v_{\boldsymbol{h}}^{\bullet} \in \mathbb{Z}_{\boldsymbol{h}}
$$

Similar definitions are given for $l\left(\mathbb{Z}_{\boldsymbol{h}}^{\bullet}\right)$ :

$$
\left\langle u_{h}^{\star}, v_{h}^{\star}\right\rangle_{l^{2}\left(\mathbb{Z}_{h}^{\star}\right)}=h^{3} \sum_{\mathbf{z} \in \mathbb{Z}_{h}^{\star}} u_{h}^{\star}(\mathbf{z}) \overline{v_{h}^{\star}}(\mathbf{z}) \quad \text { with } u_{h}^{\star}, v_{h}^{\star} \in \mathbb{Z}_{h}^{\star} .
$$

\section{Definition 4.3}

The direct restriction $R_{\boldsymbol{h}}^{\bullet}: L^{2}\left(\mathbb{R}^{3}\right) \rightarrow l\left(\mathbb{Z}_{\boldsymbol{h}}\right)$ is the operator that associates with a continuous ${ }^{7} u \in L^{2}\left(\mathbb{R}^{3}\right)$ the corresponding grid function on the grid $\mathbb{Z}_{h}$, defined

\footnotetext{
${ }^{7}$ In case of a discontinuous function we can replace $u$ by $\breve{u}$ as defined in (40).
} 
by

$$
\left(R_{\boldsymbol{h}}^{\bullet} u\right)(\boldsymbol{j} \boldsymbol{h})=u(\boldsymbol{j} \boldsymbol{h}), \quad \forall \boldsymbol{j} \in \mathbb{Z}^{3},
$$

and the direct restriction $R_{h}^{\star}: L^{2}\left(\mathbb{R}^{3}\right) \rightarrow l\left(\mathbb{Z}_{h}^{\star}\right)$ on the shifted grid $\mathbb{Z}_{h}^{\star}$ is defined by

$$
\left(R_{\boldsymbol{h}}^{\star} u\right)((\boldsymbol{j}+\mathbf{e} / 2) \boldsymbol{h})=u((\boldsymbol{j}+\mathbf{e} / 2) \boldsymbol{h}), \quad \forall \boldsymbol{j} \in \mathbb{Z}^{3} .
$$

\section{Definition 4.4}

The box restriction $R_{h}: L^{2}\left(\mathbb{R}^{3}\right) \rightarrow L^{2}\left(\mathbb{R}^{3}\right)$ is the $L^{2}$-projection on the piecewise constant functions on $\Omega_{h}$, defined by (cf. equation (6))

$$
\left(R_{h} u\right)(\boldsymbol{x})=h^{-3} \int_{\Omega_{h, j}} u(\mathbf{z}) d \mathbf{z}, \quad \forall \boldsymbol{x} \in \Omega_{h, j} .
$$

The box-restriction $R_{h}^{B}: L^{2}\left(\mathbb{R}^{3}\right) \rightarrow l\left(\mathbb{Z}_{h}^{\star}\right)$ is defined by $R_{h}^{B}=R_{h}^{\star} R_{h}$; it associates the mean value of $u$ on a cell $\Omega_{h, j}$ with the nodal value at the centre of $\Omega_{h, j}$.

The box-restriction $R_{h}^{B} u$ should be well distinguished from $R_{h}^{\star} u$. However, the $L^{2}(\Omega)$-projection $R_{h} u$ in (53) and the restriction $R_{h}^{B} u$ in (52) are conveniently related to each other by

$$
R_{h} u=\left(\frac{2 \pi}{h}\right)^{3 / 2} R_{h}^{\star}\left(\left(D_{h} \Pi\right) \star u\right) .
$$

\subsubsection{The Fourier transform of a grid function}

Let $u_{h}: \mathbb{Z}_{h} \rightarrow \mathbb{C}$ be a grid function. We give the following

\section{Definition 4.5}

The Fourier transform $\widehat{u}_{h}^{\bullet} \in L^{2}\left(T_{h}\right)$ of a grid function $u_{h}^{\bullet} \in l^{2}\left(\mathbb{Z}_{h}\right)$ is a function $T_{h} \rightarrow \mathbb{C}$, defined by

$$
\widehat{u_{\boldsymbol{h}}^{\bullet}}(\boldsymbol{\omega})=\left(\frac{h}{\sqrt{2 \pi}}\right)^{3} \sum_{\boldsymbol{j} \in \mathbb{Z}^{3}} e^{-i j h \omega} u_{\boldsymbol{h}}^{\bullet}(\boldsymbol{j h})
$$

The inverse transformation is given by

$$
u_{h}^{\bullet}(\boldsymbol{j h})=\left(\frac{1}{\sqrt{2 \pi}}\right)^{3} \int_{\omega \in T_{h}} e^{+i j \boldsymbol{h} \omega} \widehat{u_{h}^{\bullet}}(\boldsymbol{\omega}) d \boldsymbol{\omega}
$$

Let $u_{h}^{\star}: \mathbb{Z}_{h}^{\star} \rightarrow \mathbb{C}$ be a shifted grid function, then we have

\section{Definition 4.6}

The Fourier transform $\widehat{u_{h}^{\star}} \in L^{2}\left(T_{h}\right)$ of a shifted grid function $u_{h}^{\star} \in l^{2}\left(\mathbb{Z}_{h}^{\star}\right)$ is a function $T_{\boldsymbol{h}} \rightarrow \mathbb{C}$, defined by

$$
\widehat{u_{\boldsymbol{h}}^{\star}}(\boldsymbol{\omega})=\left(\frac{h}{\sqrt{2 \pi}}\right)^{3} \sum_{\boldsymbol{j} \in \mathbb{Z}^{3}} e^{-i(\boldsymbol{j}+\mathbf{e} / 2) h \omega} u_{\boldsymbol{h}}^{\star}((\boldsymbol{j}+\mathbf{e} / 2) \boldsymbol{h}) .
$$


Its inverse transformation is given by

$$
u_{h}^{*}((\boldsymbol{j}+\mathbf{e} / 2) \boldsymbol{h})=\left(\frac{1}{\sqrt{2 \pi}}\right)^{3} \int_{\boldsymbol{\omega} \in T_{h}} e^{+i(\boldsymbol{j}+\mathbf{e} / 2) \boldsymbol{h} \boldsymbol{\omega}} \hat{u_{h}^{*}}(\boldsymbol{\omega}) d \boldsymbol{\omega} .
$$

\section{Remarks}

- We immediately see that $\widehat{u_{h}^{\bullet}}(\boldsymbol{\omega})$ is $[2 \pi / \boldsymbol{h}]$-periodic, whereas $\widehat{u_{h}^{\star}}(\boldsymbol{\omega})$ is $[2 \pi / \boldsymbol{h}]$ antiperiodic, i.e. $\widehat{u_{h}^{\star}}(\boldsymbol{\omega}+2 \pi / \boldsymbol{h})=(-\mid)^{|\hat{e}|} \widehat{u_{h}^{\star}}(\boldsymbol{\omega})$.

- We denote the Fourier transforms also by

$$
\widehat{u_{h}^{*}}=\mathcal{F}\left(u_{h}^{\bullet}\right) \text { or } \quad \widehat{u_{h}^{*}}=\mathcal{F}\left(u_{h}^{\star}\right)
$$

i.e. we introduce the mapping $\mathcal{F}: l^{2}\left(\mathbb{Z}_{h}\right) \rightarrow L^{2}\left(T_{h}\right)$ or $\mathcal{F}: l^{2}\left(\mathbb{Z}_{h}^{\star}\right) \rightarrow L^{2}\left(T_{h}\right)$. At the end of this section we shall generalise this meaning of $\mathcal{F}$.

- By the Parseval equality we have

$$
\left\|u_{h}^{\bullet}\right\|_{2}=\left\|\widehat{u_{h}^{*}}\right\|_{L^{2}\left(T_{h}\right)} \text { and }\left\|u_{h}^{\star}\right\|_{2}=\left\|\widehat{u_{h}^{\star}}\right\|_{L^{2}\left(T_{h}\right)} \text {. }
$$

Hence the Fourier transformation operators $\mathcal{F}: l^{2}\left(\mathbb{Z}_{h}\right) \rightarrow L^{2}\left(T_{h}\right)$ and $\mathcal{F}: l^{2}\left(\mathbb{Z}_{h}^{\star}\right) \rightarrow L^{2}\left(T_{h}\right)$ are unitary operators.

- Because $e_{\boldsymbol{h}, \omega}^{\bullet} \equiv e_{\boldsymbol{h}, \omega+2 \pi k / \boldsymbol{h}}^{\bullet}$ or $e_{\boldsymbol{h}, \omega}^{\star} \equiv(-)^{|\boldsymbol{e} k|} e_{h, \omega+2 \pi k / \boldsymbol{h}}^{\star}$, for all $\boldsymbol{k} \in \mathbb{Z}^{3}$, on a mesh of size $\boldsymbol{h}$ a frequency $\boldsymbol{\omega}$ cannot be distinguished from a frequency $\boldsymbol{\omega}+2 \pi \boldsymbol{k} / \boldsymbol{h}$. This phenomenon is called aliasing.

\subsubsection{The relation between FTs of a function restricted to different grids}

In this section we first present a few theorems associated with the different restrictions between two grids. We describe the relation between the FT of a continuous function defined on $\mathbb{R}^{3}$ and the FT of its restriction to the grid and then we show the relation between the FT of a fine grid function and the FT of its representation on a coarser grid. Next, we give the corresponding theorems for the prolongations.

\section{Lemma 4.7}

Let $u \in L^{2}\left(\mathbb{R}^{3}\right)$ be a continuous function with FT $\hat{u}$. Its restriction $u_{h}^{\bullet}$ to the grid $\mathbb{Z}_{h}$ is defined by (51). We have the following relation between $\hat{u}$ and $\widehat{u_{h}^{\circ}}$ :

$$
\widehat{u_{h}^{*}}(\boldsymbol{\omega})=\sum_{\boldsymbol{k} \in \mathbb{Z}^{3}} \hat{u}(\boldsymbol{\omega}+2 \pi \boldsymbol{k} / \boldsymbol{h})
$$

i.e. $\widehat{u_{\boldsymbol{h}}^{\bullet}}$ is the $[2 \pi / \boldsymbol{h}]$-periodisation of $\hat{u}$.

Proof

For the proof we refer to [4].

In the following lemmas $\mathbf{q}$-restrictions are considered, with $\mathbf{q} \in \mathbb{Z}^{3}$. Here $\mathbf{q}=\left(q_{1}, q_{2}, q_{3}\right)$ is the coarsening factor, where usually $1 \leqslant q_{j} \leqslant 2, j=1,2,3$. 


\section{Definition 4.8}

Let $\mathbf{q} \in \mathbb{Z}^{3}$ with $\mathbf{q}>\mathbf{0}$ and $\boldsymbol{H}=\mathbf{q} \boldsymbol{h}$, then the canonical $\mathbf{q}$-restriction $R_{\mathbf{q}}^{\bullet}$ is the operator $R_{\mathbf{q}}^{\bullet}: l\left(\mathbb{Z}_{\boldsymbol{h}}\right) \rightarrow l\left(\mathbb{Z}_{\mathbf{q} h}\right)=l\left(\mathbb{Z}_{\boldsymbol{H}}\right)$, defined by

$$
\left(R_{\mathrm{q}}^{\bullet} u_{\boldsymbol{h}}^{\bullet}\right)(\boldsymbol{j} \boldsymbol{H})=u_{\boldsymbol{H}}^{\bullet}(\mathbf{j H})=u_{\boldsymbol{h}}^{\bullet}(\mathbf{j q} \boldsymbol{h}) .
$$

\section{Theorem 4.9}

We have the following relation between the FT of a grid function and that of its canonical q-restriction,

$$
\left(\widehat{R_{\mathbf{q}}^{\bullet} u_{\boldsymbol{h}}^{\bullet}}\right)(\boldsymbol{\omega})=\sum_{\boldsymbol{p} \in[0, \mathbf{q})} \widehat{u_{\boldsymbol{h}}^{\bullet}}(\boldsymbol{\omega}+2 \pi \boldsymbol{p} / \boldsymbol{h}), \quad \forall \boldsymbol{\omega} \in T_{\boldsymbol{H}}, \boldsymbol{H}=\mathbf{q} \boldsymbol{h}
$$

\section{Proof}

For the proof we refer to [4].

Lemma 4.9 shows that, using the restriction $R_{\mathbf{q}}^{\bullet}$ with $\mathbf{q} \in \mathbb{Z}^{3}, \mathbf{q}>\mathbf{0}$, we get aliasing of $q^{3}=q_{1} \cdot q_{2} \cdot q_{3}$ frequencies onto one.

Now we describe the relation between the Fourier transforms of a continuous function and its box restrictions. First we consider the direct restriction to the shifted grid, $R_{h}^{\star}$, and later the box-restriction, $R_{h}$ and the q-restriction $R_{\mathbf{q}}^{\star}$.

\section{Lemma 4.10}

$$
\widehat{u_{h}^{\star}}(\boldsymbol{\omega})=\widehat{R_{h}^{\star} u}(\omega)=\sum_{\boldsymbol{k} \in \mathbb{Z}^{3}}(-)^{|k|} \hat{u}(\boldsymbol{\omega}+2 \pi \boldsymbol{k} / \boldsymbol{h})
$$

Proof

For the proof we refer to [4].

For the Fourier transform of $u \in L^{2}\left(\mathbb{R}^{3}\right)$ and $R_{h} u \in L^{2}\left(\mathbb{R}^{3}\right)$ we have the following relation.

\section{Theorem 4.11}

$$
\widehat{u_{h}}(\boldsymbol{\omega})=\widehat{R_{h} u}(\boldsymbol{\omega})=\sum_{\boldsymbol{k} \in \mathbb{Z}^{3}}(-)^{|\boldsymbol{k}|} \operatorname{Sinc}\left(\frac{\boldsymbol{h} \boldsymbol{\omega}}{2 \pi}+\boldsymbol{k}\right) \cdot \hat{u}(\boldsymbol{\omega}+2 \pi \boldsymbol{k} / \boldsymbol{h}) .
$$

Proof

Using (54) we see

$$
\begin{aligned}
\widehat{u_{h}^{\star}}(\boldsymbol{\omega}) & =\mathcal{F}\left(R_{h} u\right)(\boldsymbol{\omega}) \\
& =\left(\frac{2 \pi}{h}\right)^{3 / 2} \mathcal{F}\left(R_{\boldsymbol{h}}^{\star}\left(\left(D_{\boldsymbol{h}} \Pi\right) \star u\right)\right)(\boldsymbol{\omega}) \\
& =\left(\frac{2 \pi}{h}\right)^{3 / 2} \sum_{\boldsymbol{k}}(-)^{|\boldsymbol{k}|} \mathcal{F}\left(\left(D_{h} \Pi\right) \star u\right)(\boldsymbol{\omega}+2 \pi \boldsymbol{k} / \boldsymbol{h})
\end{aligned}
$$




$$
\begin{aligned}
& =\left(\frac{2 \pi}{h}\right)^{3 / 2} \sum_{\boldsymbol{k}}(-)^{|\boldsymbol{k}|}\left(\widehat{D_{\boldsymbol{h}} \Pi}\right)(\boldsymbol{\omega}+2 \pi \boldsymbol{k} / \boldsymbol{h}) \cdot \hat{u}(\boldsymbol{\omega}+2 \pi \boldsymbol{k} / \boldsymbol{h}) \\
& =\left(\frac{2 \pi}{h}\right)^{3 / 2} \sum_{\boldsymbol{k}}(-)^{|\boldsymbol{k}|} D_{1 / \boldsymbol{h}} \hat{\Pi}(\boldsymbol{\omega}+2 \pi \boldsymbol{k} / \boldsymbol{h}) \cdot \hat{u}(\boldsymbol{\omega}+2 \pi \boldsymbol{k} / \boldsymbol{h}) . \\
& =h^{-3 / 2} \sum_{\boldsymbol{k}}(-)^{|\boldsymbol{k}|} D_{1 / \boldsymbol{h}} \operatorname{Sinc}\left(\frac{\omega}{2 \pi}=\boldsymbol{k} / \boldsymbol{h}\right) \cdot \hat{u}(\boldsymbol{\omega}+2 \pi \boldsymbol{k} / \boldsymbol{h}) . \\
& =\sum_{\boldsymbol{k}}(-)^{|\boldsymbol{k}|} \operatorname{Sinc}\left(\frac{\boldsymbol{h} \boldsymbol{\omega}}{2 \pi}+\boldsymbol{k}\right) \cdot \hat{u}(\boldsymbol{\omega}+2 \pi \boldsymbol{k} / \boldsymbol{h}) .
\end{aligned}
$$

\section{Definition 4.12}

Let $\mathbf{q} \in \mathbb{Z}^{3}$ with $\mathbf{q}>\mathbf{0}$ and $\boldsymbol{H}=\mathbf{q} \boldsymbol{h}$, then, for $\mathbf{s} \in[\mathbf{o}, \mathbf{q})$, the s-frequency decomposition q-restriction is the operator $R_{\mathbf{q}}^{\mathrm{s}}: l\left(\mathbb{Z}_{\boldsymbol{h}}\right) \rightarrow l\left(\mathbb{Z}_{\mathbf{q} h}\right)=l\left(\mathbb{Z}_{\boldsymbol{H}}\right)$ is defined by

$$
\left(R_{\mathbf{q}}^{\mathbf{s}} u_{h}^{\star}\right)((\boldsymbol{j}+\mathbf{e} / 2) \boldsymbol{H})=q^{-3} \sum_{\boldsymbol{k} \in[\mathbf{0}, \mathbf{q})}(-)^{\mathbf{s} k} u_{\boldsymbol{h}}^{\star}((\mathbf{q} \boldsymbol{j}+\boldsymbol{k}+\mathbf{e} / 2) \boldsymbol{h}),
$$

where $q^{3}$ denotes $q^{3}=q_{1} \cdot q_{2} \cdot q_{3}$.

\section{Remarks}

- In the case $\mathbf{s}=\mathbf{o}$ we call $R_{\mathrm{q}}^{\mathbf{s}}=R_{\mathbf{q}}^{\mathbf{0}}=R_{\mathbf{q}}^{\star}$ simply the q-restriction.

- From the construction of the restriction operators $R_{h}^{B}$ and $R_{\mathbf{q}}^{\star}$ it is clear that the following relation holds:

$$
R_{H}^{B}=R_{\mathbf{q}}^{\star} R_{h}^{B}
$$

\section{Theorem 4.13}

Let $\mathbf{q}=2 \mathrm{e} \in \mathbb{Z}^{3}$, then for all $\boldsymbol{\omega} \in T_{\boldsymbol{H}}^{3}, \boldsymbol{H}=2 \boldsymbol{h}$, we have

$$
\left(\widehat{R_{\mathbf{q}}^{\mathbf{s}} u_{\boldsymbol{h}}^{\star}}\right)(\boldsymbol{\omega})=2 q^{-3} i^{\mathbf{s}} \sum_{\mathbf{m} \in[\mathbf{o}, \mathbf{q})}(-)^{\mathbf{m}} \operatorname{Cos}\left(\frac{\boldsymbol{h} \boldsymbol{\omega}+\pi(\mathbf{m}+\mathbf{s})}{2}\right) \cdot \widehat{u_{\boldsymbol{h}}^{\star}}(\boldsymbol{\omega}+\pi \mathbf{m} / \boldsymbol{h}),
$$

where

$$
\operatorname{Cos}(\boldsymbol{h} \boldsymbol{\omega} / 2)=\prod_{j=1,2,3} \cos \left(h_{j} \omega_{j} / 2\right)
$$

\section{Proof}

For the proof we refer to [4].

\section{Definition 4.14}

The natural box-prolongation $P_{h}^{\star}: l^{2}\left(\mathbb{Z}_{h}^{\star}\right) \rightarrow L^{2}\left(\mathbb{R}^{3}\right)$ is defined by

$$
u(\boldsymbol{x})=\left(P_{h}^{\star} u_{h}^{\star}\right)(\boldsymbol{x})=u_{h}((\mathbf{m}+\mathbf{e} / 2) \boldsymbol{h}),
$$


for all $\mathbf{m} \in \mathbb{Z}^{3}$ and $\mathbf{m} \boldsymbol{h}<\boldsymbol{x}<(\mathbf{m}+\mathbf{e}) \boldsymbol{h}$. We also introduce a natural prolongation, $P_{\mathrm{q}}^{\star}$, from a coarse to a fine gridfunction $P_{\mathrm{q}}^{\star}: l^{2}\left(\mathbb{Z}_{H}^{\star}\right) \rightarrow l^{2}\left(\mathbb{Z}_{h}^{\star}\right)$ by $P_{\mathrm{q}}^{\star}=R_{h}^{\star} P_{H}^{\star}$ where $\boldsymbol{H}=\mathbf{q} \boldsymbol{h}$. The prolongation $P_{h}^{B}: l\left(\mathbb{Z}_{h}^{*}\right) \rightarrow L^{2}\left(\mathbb{R}^{3}\right)$ is the operator dual to $R_{h}^{B}$ in the sense that for all $u_{h}^{\star} \in l^{2}\left(\mathbb{Z}_{h}^{\star}\right)$ and $v \in L^{2}\left(\mathbb{R}^{3}\right)$ we have

$$
\left\langle P_{h}^{B} u_{h}^{\star}, v\right\rangle_{L^{2}\left(\mathbb{R}^{3}\right)}=\left\langle u_{h}^{\star}, R_{h}^{B} v\right\rangle_{l^{2}\left(\mathbb{Z}_{h}^{\star}\right)} .
$$

The following theorems show how we find the FT of the prolongation if the FT of the source function is given.

\section{Lemma 4.15}

$$
\hat{u}(\omega)=\mathcal{F}\left(P_{h}^{\star} u_{h}^{\star}\right)(\omega)=\frac{\operatorname{Sin}(\boldsymbol{h} \boldsymbol{\omega} / 2)}{\boldsymbol{h} \boldsymbol{\omega} / 2} \widehat{u_{h}^{\star}}(\boldsymbol{\omega})
$$

Proof

For the proof we refer to [4].

\section{Theorem 4.16}

With $\boldsymbol{H}=\boldsymbol{q} \boldsymbol{h}$ and $q^{3}=q_{1} \cdot q_{2} \cdot q_{3}$, we have for the FT of the prolongation of a box gridfunction

$$
\widehat{u_{h}^{\star}}(\boldsymbol{\omega})=\mathcal{F}\left(P_{\mathbf{q}}^{\star} u_{H}^{\star}\right)(\boldsymbol{\omega})=q^{-3} \frac{\operatorname{Sin}(\boldsymbol{H} \boldsymbol{\omega} / 2)}{\operatorname{Sin}(\boldsymbol{h} \boldsymbol{\omega} / 2)} \widehat{u_{\boldsymbol{H}}^{\star}}(\boldsymbol{\omega})
$$

\section{Proof}

For the proof we refer to [4].

\subsubsection{The Fourier transform of operators involving different grids}

In (68) and (71) we see that, by the restriction and prolongation between functions on grids $\Omega_{\mathbf{n}}$ and $\Omega_{\mathbf{n}+\mathbf{q}}$, aliasing takes place and that $q^{3}$ frequencies on $\Omega_{\mathbf{n}+\mathbf{q}}$ correspond with a single frequency on $\Omega_{\mathrm{n}}$. This implies that, analysing a multigrid algorithm, we have to study the behaviour of the $q^{3}$ aliasing frequencies together. Collecting the $q^{3}$ corresponding amplitudes of the aliasing frequencies in a single $q^{3}$ vector, we extend the definition (59) of $\mathcal{F}$ to the case $q^{3}>1$ and obtain $\mathcal{F}: l^{2}\left(\mathbb{Z}_{h}\right) \rightarrow$ $\left[L^{2}\left(T_{\mathbf{q} h}\right)\right]^{q^{3}}$ or $\mathcal{F}: l^{2}\left(\mathbb{Z}_{h}^{*}\right) \rightarrow\left[L^{2}\left(T_{\mathbf{q} h}\right)\right]^{q^{3}}$ by

$$
\mathcal{F}\left(u_{\boldsymbol{h}}\right)(\boldsymbol{\omega})=\left(\hat{u}_{\boldsymbol{h}}(\boldsymbol{\omega}+\pi \mathbf{m} / \boldsymbol{h})\right)_{\mathbf{m} \in[\mathbf{0}, \mathbf{q})}, \quad \boldsymbol{\omega} \in T_{\mathbf{q} h} .
$$

With these amplitude-vectors $\mathcal{F}\left(u_{h}^{*}\right)(\omega)$ and $\mathcal{F}\left(u_{h}^{\star}\right)(\omega)$, we can introduce the linear operators $\mathcal{F}\left(R_{\mathbf{q}}^{*}\right)(\boldsymbol{\omega})$ and $\mathcal{F}\left(R_{\mathbf{q}}^{\star}\right)(\boldsymbol{\omega})$ by

$$
\mathcal{F}\left(R_{\mathbf{q}}^{\bullet} u_{\boldsymbol{h}}^{\bullet}\right)(\boldsymbol{\omega})=\mathcal{F}\left(R_{\mathbf{q}}^{\bullet}\right)(\boldsymbol{\omega}) \mathcal{F}\left(u_{\boldsymbol{h}}^{\bullet}\right)(\boldsymbol{\omega}), \quad \boldsymbol{\omega} \in T_{\mathrm{q} h},
$$

and

$$
\mathcal{F}\left(R_{\mathbf{q}}^{\star} u_{h}^{\star}\right)(\boldsymbol{\omega})=\mathcal{F}\left(R_{\mathbf{q}}^{\star}\right)(\boldsymbol{\omega}) \mathcal{F}\left(u_{h}^{\star}\right)(\boldsymbol{\omega}), \quad \boldsymbol{\omega} \in T_{\mathbf{q} h} .
$$

We call the new operators, that depend on $\omega$, the Fourier transforms of the original 
operators. The new operators are $\ell \times q^{3} \ell$ matrices if $\ell$ aliasing frequencies are considered on the coarse grid.

Similar to the restrictions, the prolongations can be associated with their Fourier transforms.

$$
\mathcal{F}\left(P_{\mathbf{q}}^{\bullet} u_{\boldsymbol{h}}^{\bullet}\right)(\boldsymbol{\omega})=\mathcal{F}\left(P_{\mathbf{q}}^{\bullet}\right)(\boldsymbol{\omega}) \mathcal{F}\left(u_{\boldsymbol{h}}^{\bullet}\right)(\boldsymbol{\omega}), \quad \boldsymbol{\omega} \in T_{\mathbf{q} h},
$$

and

$$
\mathcal{F}\left(P_{\mathbf{q}}^{\star} u_{h}^{\star}\right)(\boldsymbol{\omega})=\mathcal{F}\left(P_{\mathbf{q}}^{\star}\right)(\boldsymbol{\omega}) \mathcal{F}\left(u_{h}^{\star}\right)(\boldsymbol{\omega}), \quad \boldsymbol{\omega} \in T_{\mathbf{q} h},
$$

These operators are $q^{3} \ell \times \ell$ matrices.

For arbitrary linear constant coefficient operators $A_{h}: l^{2}\left(\mathbb{Z}_{h}\right) \rightarrow l^{2}\left(\mathbb{Z}_{h}\right)$, its Fourier transform $\mathcal{F}\left(A_{h}\right): L^{2}\left(T_{h}\right) \rightarrow L^{2}\left(T_{h}\right)$, can also be considered as a $q^{3} \ell \times q^{3} \ell$ diagonal matrix

$$
\mathcal{F}\left(A_{h} u_{h}\right)(\omega)=\mathcal{F}\left(A_{h}\right)(\omega) \cdot \mathcal{F}\left(u_{h}\right)(\boldsymbol{\omega}), \quad \forall \boldsymbol{\omega} \in T_{\mathbf{q} h}
$$

Because of Parseval's equality we know that

$$
\left\|A_{\boldsymbol{h}}\right\|_{2}=\max _{\boldsymbol{\omega} \in T_{\mathbf{q} h}}\left\|\mathcal{F}\left(A_{\boldsymbol{h}}\right)(\boldsymbol{\omega})\right\|_{2}=\max _{\boldsymbol{\omega} \in T_{\mathbf{q} h}} \sigma\left(\mathcal{F}\left(A_{\boldsymbol{h}}\right)\right)(\boldsymbol{\omega}),
$$

with $\sigma(A)$ the spectral norm (the largest singular value) of the matrix $A$, and

$$
\rho\left(A_{\boldsymbol{h}}\right)=\max _{\boldsymbol{\omega} \in T_{\mathbf{q}^{h}}} \rho\left(\mathcal{F}\left(A_{\boldsymbol{h}}\right)\right)(\boldsymbol{\omega}),
$$

where $\rho$ denotes the spectral radius. This provides us with the means to study the norm and the spectral radius of the error-amplification operator of the multigrid iteration.

\subsection{Fourier analysis convergence results}

To get some insight in the behaviour of the more-dimensional multigrid algorithm introduced in section 3, we use the Fourier analysis to determine the convergence rate of the two-level algorithm for the two-dimensional anisotropic Poisson equation

$$
u_{x x}+\epsilon^{2} u_{y y}=f
$$

discretised by the usual 5 -point discretisation.

The error-amplification operator, $M_{\mathrm{n}}$, of the two-level cycle (with $\mu$ pre- and $\nu$ post-relaxation steps) for the solution of (29) is described by

$$
e_{\mathbf{n}}^{(i+1)}=M_{\mathbf{n}} e_{\mathbf{n}}^{(i)}=S_{\mathbf{n}}^{\nu} C_{\mathbf{n}} S_{\mathbf{n}}^{\mu} e_{\mathbf{n}}^{(i)}
$$

where $S_{\mathrm{n}}$ denotes the smoothing, e.g. damped Jacobi iteration:

$$
e_{\mathbf{n}}^{\text {(new) }}=S_{\mathbf{n}} e_{\mathbf{n}}^{\text {(old) }}=\left(I_{\mathbf{n}}-\alpha D_{\mathbf{n}}^{-1} L_{\mathbf{n}}\right) e_{\mathbf{n}}^{\text {(old) }},
$$

with $\alpha$ the damping parameter and $D_{\mathbf{n}}$ the main diagonal of the discrete operator $L_{\mathbf{n}}$. The coarse grid correction is described by (30) and (31). For gridfunctions 
$u_{h} \in l^{2}\left(\mathbb{Z}_{h}^{\star}\right)$, i.e. neglecting boundary conditions, we find, using (30)

$$
\mathcal{F}\left(M_{\mathbf{n}}\right)(\boldsymbol{\omega})=\mathcal{F}\left(S_{\mathbf{n}}\right)^{\nu}(\boldsymbol{\omega}) \mathcal{F}\left(C_{\mathbf{n}}\right)(\boldsymbol{\omega}) \mathcal{F}\left(S_{\mathbf{n}}\right)^{\mu}(\boldsymbol{\omega}),
$$

with

$$
\mathcal{F}\left(S_{\mathbf{n}}\right)=\mathcal{F}\left(I_{\mathbf{n}}\right)-\alpha \mathcal{F}\left(D_{\mathbf{n}}\right)^{-1} \mathcal{F}\left(L_{\mathbf{n}}\right)
$$

and

$$
\begin{aligned}
\mathcal{F}\left(C_{\mathbf{n}}\right)= & \mathcal{F}\left(I_{\mathbf{n}}\right) \\
& -\sum_{j=1,2,3} \mathcal{F}\left(P_{\mathbf{n}, \mathbf{n}-\mathbf{e}_{j}}\right) \mathcal{F}\left(L_{\mathbf{n}-\mathbf{e}_{j}}\right)^{-1} \mathcal{F}\left(R_{\mathbf{n}-\mathbf{e}_{j}, \mathbf{n}}\right) \mathcal{F}\left(L_{\mathbf{n}}\right) \\
& +\sum_{j=1,2,3} \mathcal{F}\left(P_{\mathbf{n}, \mathbf{n}-\mathbf{e}+\mathbf{e}_{j}}\right) \mathcal{F}\left(L_{\mathbf{n}-\mathbf{e}+\mathbf{e}_{j}}\right)^{-1} \mathcal{F}\left(R_{\mathbf{n}-\mathbf{e}+\mathbf{e}_{j}, \mathbf{n}}\right) \mathcal{F}\left(L_{\mathbf{n}}\right) \\
& -\mathcal{F}\left(P_{\mathbf{n}, \mathbf{n}-\mathbf{e}}\right) \mathcal{F}\left(L_{\mathbf{n}-\mathbf{e}}\right)^{-1} \mathcal{F}\left(R_{\mathbf{n}-\mathbf{e}, \mathbf{n}}\right) \mathcal{F}\left(L_{\mathbf{n}}\right) .
\end{aligned}
$$

To get an impression of the behaviour of the algorithm, keeping the explicit computation to reasonable size, we elaborate (82) for the equation (79), for the two-dimensional case, with $\mathbf{q}=(2,2)$ and $\mu=\nu=1$. Then $\mathcal{F}\left(M_{\mathbf{n}}\right)(\boldsymbol{\omega})$ is a $4 \times 4$ matrix, which we derive from (82), (83) and (cf. (15))

$$
\begin{aligned}
\mathcal{F}\left(C_{\mathbf{n}}\right)= & \mathcal{F}\left(I_{\mathbf{n}}\right) \\
& -\mathcal{F}\left(P_{\mathbf{n}, \mathbf{n}-\mathbf{e}_{1}}\right) \mathcal{F}\left(L_{\mathbf{n}-\mathbf{e}_{1}}\right)^{-1} \mathcal{F}\left(R_{\mathbf{n}-\mathbf{e}_{1}, \mathbf{n}}\right) \mathcal{F}\left(L_{\mathbf{n}}\right) \\
& -\mathcal{F}\left(P_{\mathbf{n}, \mathbf{n}-\mathbf{e}_{2}}\right) \mathcal{F}\left(L_{\mathbf{n}-\mathbf{e}_{2}}\right)^{-1} \mathcal{F}\left(R_{\mathbf{n}-\mathbf{e}_{2}, \mathbf{n}}\right) \mathcal{F}\left(L_{\mathbf{n}}\right) \\
& +\mathcal{F}\left(P_{\mathbf{n}, \mathbf{n}-\mathbf{e}}\right) \mathcal{F}\left(L_{\mathbf{n}-\mathbf{e}}\right)^{-1} \mathcal{F}\left(R_{\mathbf{n}-\mathbf{e}, \mathbf{n}}\right) \mathcal{F}\left(L_{\mathbf{n}}\right) .
\end{aligned}
$$

From (68) and (71) we know

$$
\begin{aligned}
& \mathcal{F}\left(R_{\mathbf{n}-\mathbf{e}_{1}, \mathbf{n}}\right)=\left(\begin{array}{cccc}
\cos \omega_{2} h_{2} & 0 & \sin \omega_{2} h_{2} & 0 \\
0 & \cos \omega_{2} h_{2} & 0 & \sin \omega_{2} h_{2}
\end{array}\right), \\
& \mathcal{F}\left(R_{\mathbf{n}-\mathbf{e}_{2}, \mathbf{n}}\right)=\left(\begin{array}{cccc}
\cos \omega_{1} h_{1} & \sin \omega_{1} h_{1} & 0 & 0 \\
0 & 0 & \cos \omega_{1} h_{2} & \sin \omega_{1} h_{1}
\end{array}\right)
\end{aligned}
$$

and

$$
\mathcal{F}\left(R_{\mathbf{n}-\mathbf{e}, \mathbf{n}}\right)=\left(\begin{array}{c}
\cos \left(\omega_{1} h_{1}\right) \cos \left(\omega_{2} h_{2}\right) \\
\sin \left(\omega_{1} h_{1}\right) \cos \left(\omega_{2} h_{2}\right) \\
\cos \left(\omega_{1} h_{1}\right) \sin \left(\omega_{2} h_{2}\right) \\
\sin \left(\omega_{1} h_{1}\right) \sin \left(\omega_{2} h_{2}\right)
\end{array}\right)^{T} .
$$

So, with $\mathcal{F}\left(P_{\mathbf{n}, \mathbf{n}-\mathbf{e}_{1}}\right)=\mathcal{F}\left(R_{\mathbf{n}-\mathbf{e}_{1}, \mathbf{n}}\right)^{T}, \mathcal{F}\left(P_{\mathbf{n}, \mathbf{n}-\mathbf{e}_{2}}\right)=\mathcal{F}\left(R_{\mathbf{n}-\mathbf{e}_{2}, \mathbf{n}}\right)^{T}$ and $\mathcal{F}\left(P_{\mathbf{n}, \mathbf{n}-\mathbf{e}}\right)=$ $\mathcal{F}\left(R_{\mathbf{n}-\mathbf{e}, \mathbf{n}}\right)^{T}$, the norm $\left\|M_{\mathbf{n}}\right\|$ and the spectral radius $\rho\left(M_{\mathbf{n}}\right)$ can be computed by means of (77) and (78). 


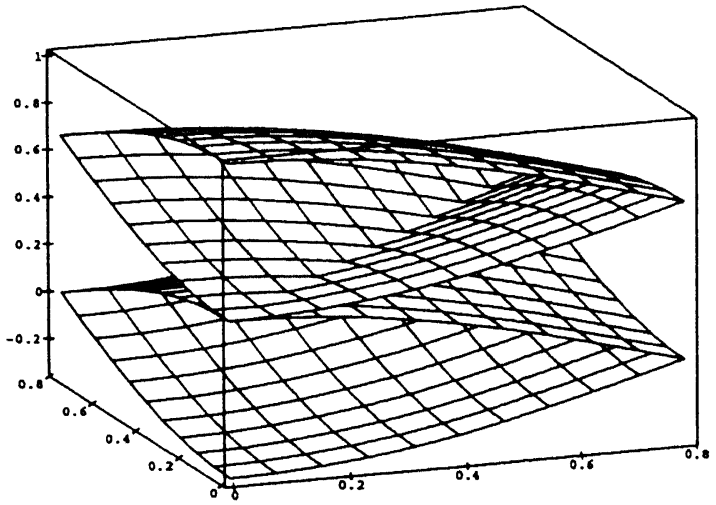

(a) The eigenvalues of $\mathcal{F}\left(S_{\mathbf{n}}\right)(\boldsymbol{\omega})$.

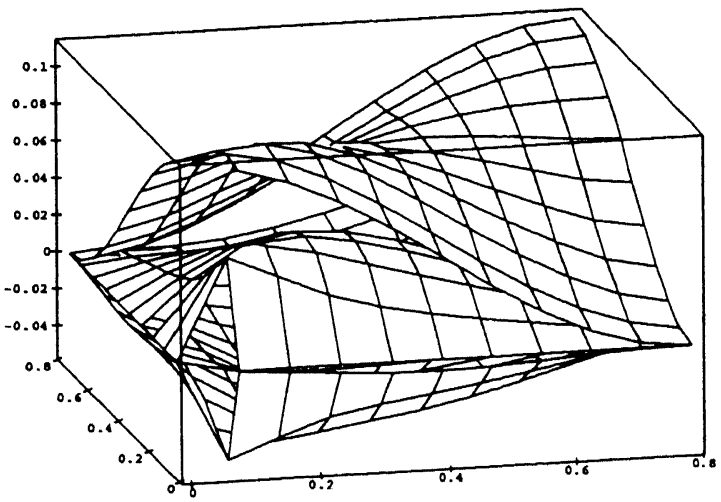

(c) The eigenvalues of $\mathcal{F}\left(M_{\mathbf{n}}\right)(\boldsymbol{\omega})$

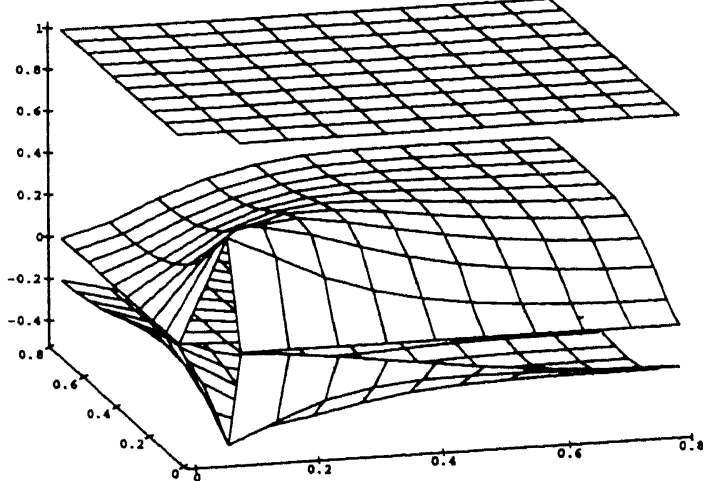

(b) The eigenvalues of $\mathcal{F}\left(C_{\mathbf{n}}\right)(\boldsymbol{\omega})$.

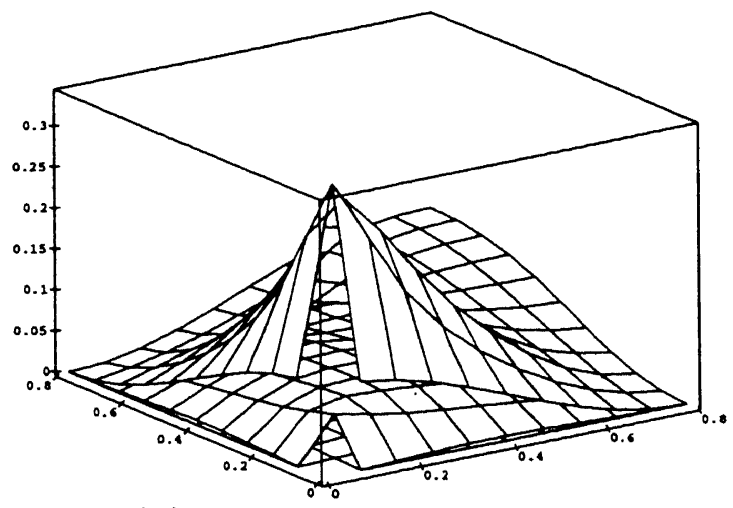

(d) The singular values of $\mathcal{F}\left(M_{\mathbf{n}}\right)(\boldsymbol{\omega})$.

Figure 1. The frequency response of the operators $S_{\mathbf{n}}, C_{\mathbf{n}}$ and $M_{\mathbf{n}}$, for $\epsilon=1, \mathbf{q}=(2.2)$ and $\alpha=2 / 3$.

To study the convergence behaviour of our algorithm, we consider the matrices (83), (84) and (82) as a function of $\boldsymbol{\omega} \in T_{\boldsymbol{h}}=[-\pi / h, \pi / h]^{2}$, and for each $\boldsymbol{\omega}$ we compute the eigenvalues and singular values of these matrices. We show these values in figure 1 for the case $\alpha=2 / 3, \epsilon=1$. Without loss of generality we can take $\boldsymbol{h}=(1,1)$, the parameter $\epsilon$ taking care of the anisotropy. The damping parameter $\alpha \in[0,1]$ for the Jacobi relaxation can be chosen freely. We select the value $\alpha=2 / 3$ because it minimises

$$
\max _{\omega=(0, \pi / h),(\phi, 0),(\pi / h, \pi / h)} \rho\left(\mathcal{F}\left(S_{\mathbf{n}}\right)(\boldsymbol{\omega})\right) .
$$

This means that $\alpha=2 / 3$ makes $S_{\mathbf{n}}$ a well balanced smoother for the different types of high frequencies (see figure 1a).

In figure 1a we show the eigenvalues of the smoothing operator, and in figure $1 \mathrm{~b}$ of the coarse grid correction. In this figure we see that one eigenvalue of $\mathcal{F}\left(C_{\mathbf{n}}\right)$ is always equal to one. This eigenvalue corresponds with the highest frequencies, for which no correction can be obtained from any of the three coarser grids. The combined effect of the smoother and the coarse grid correction is seen in figure $1 \mathrm{c}$, which shows that $\sup _{\omega} \rho\left(M_{\mathbf{n}}(\omega)\right) \approx 1 / 9$, and also in figure $1 \mathrm{~d}$, where we see 


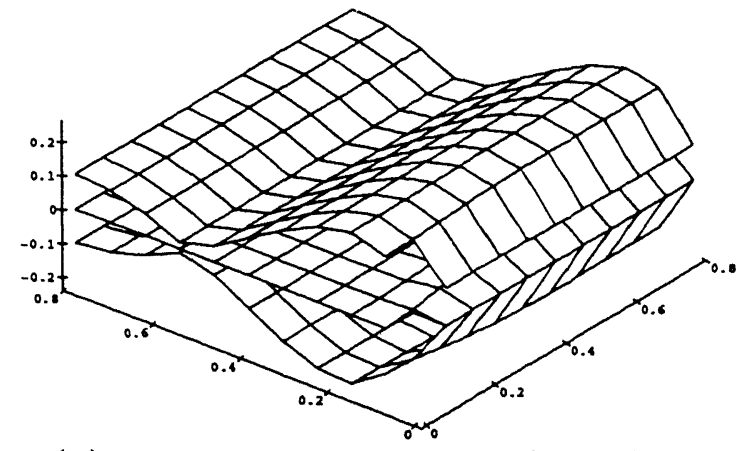

(a) The eigenvalues of $\mathcal{F}\left(M_{\mathbf{n}}\right)(\boldsymbol{\omega})$.

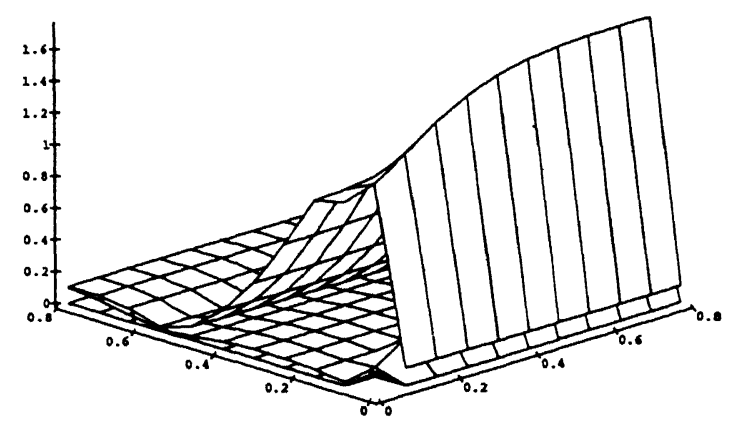

(b) The singular values of $\mathcal{F}\left(M_{\mathbf{n}}\right)(\boldsymbol{\omega})$.

Figure 2. The frequency response of the operator $M_{\mathbf{n}}$, for $\epsilon=1 / 8, \mathbf{q}=(2,2)$ and $\alpha=2 / 3$.

$\sup _{\boldsymbol{\omega}}\left\|M_{\mathbf{n}}(\boldsymbol{\omega})\right\| \approx 1 / 3$. The rather low maximal values show that - at least for square fine-grid cells - the multigrid algorithm has good convergence behaviour.

Because it is important that the algorithm is effective for an arbitrary cell aspect ratio, in figures 2-4 we show the singular values of $\mathcal{F}\left(M_{\mathbf{n}}\right)(\boldsymbol{\omega})$ also for $\epsilon=1 / 8$ and for the limit as $\epsilon \rightarrow 0$. Now it appears that for high aspect ratios the convergence behaviour deteriorates. We find $\sup _{\omega} \lim _{\epsilon \rightarrow 0} \sigma\left(\mathcal{F}\left(M_{\mathbf{n}}\right)(\omega)\right) \approx 5$. This implies that we cannot always guarantee error reduction for a single iteration sweep. Therefore we show in figure 4 also the behaviour of $M_{\mathbf{n}}^{2}(\boldsymbol{\omega})$. This shows that a couple of two consecutive iteration steps does guarantee error reduction, and the convergence rate is significant:

$$
\sup _{\boldsymbol{\omega}} \lim _{\epsilon \rightarrow 0} \rho\left(\mathcal{F}\left(M_{\mathbf{n}}^{2}\right)(\boldsymbol{\omega})\right) \approx 1 / 9
$$

As a consequence we can expect that a W-type cycle of the multigrid algorithm will have good convergence properties.

From the computations of which the results are summarised in figures 2-4, we conclude that the eigenvalues of the iteration matrix are less than 1 , uniformly in the parameter $\epsilon$. In fact, $\max _{\epsilon} \rho\left(M_{\mathbf{n}}\right) \approx 0.33$ and $\max _{\epsilon}\left\|M_{\mathbf{n}}\right\| \approx 5.0$ and

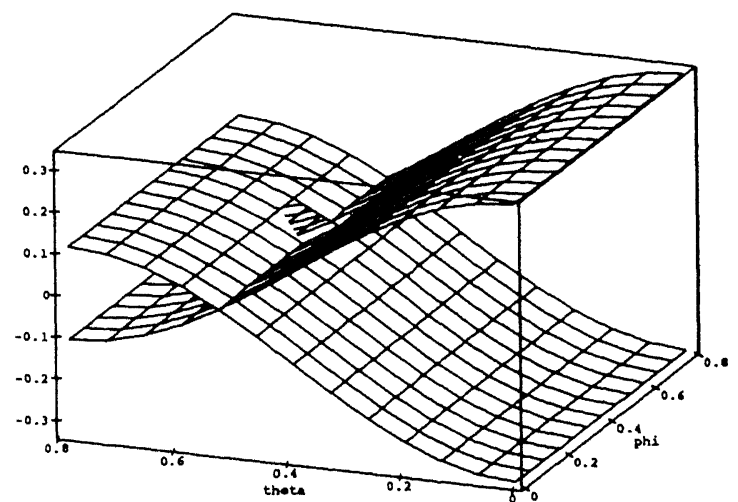

(a) The eigenvalues of $\lim _{\varepsilon \rightarrow 0} \mathcal{F}\left(M_{\mathbf{n}}\right)(\boldsymbol{\omega})$.

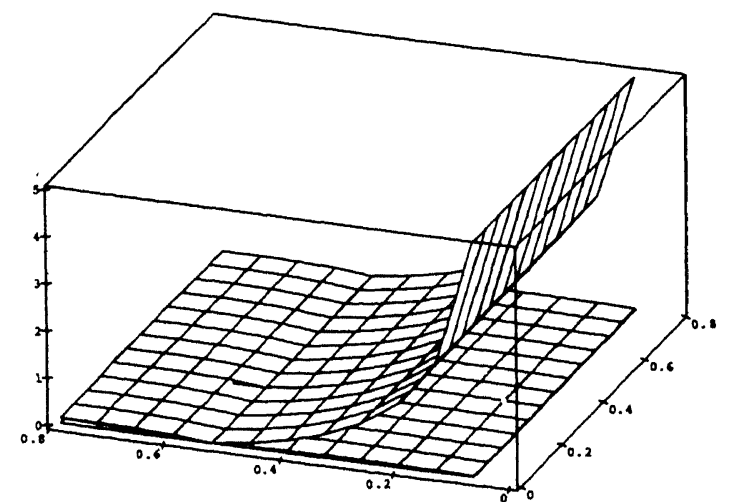

(b) The singular values of $\lim _{\varepsilon \rightarrow 0} \mathcal{F}\left(M_{\mathbf{n}}\right)(\boldsymbol{\omega})$.

Figure 3. The frequency response of the operator $\lim _{\epsilon \rightarrow 0} M_{\mathbf{n}}$, for $\mathbf{q}=(2,2)$ and $\alpha=2 / 3$. 


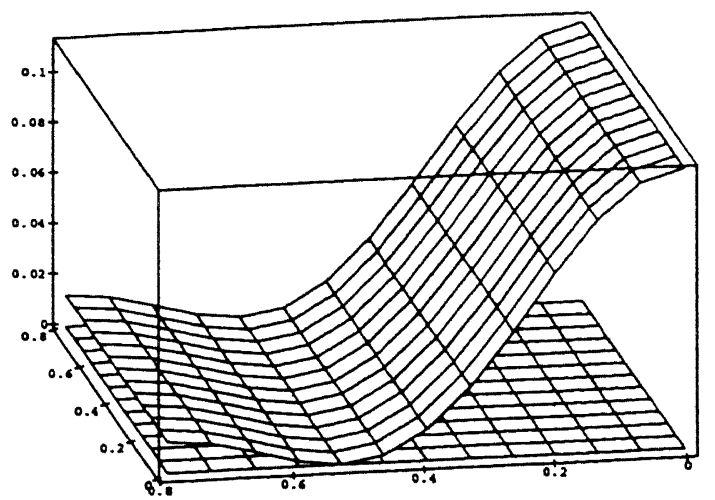

(a) The eigenvalues of $\lim _{\varepsilon \rightarrow 0} \mathcal{F}\left(M_{\mathbf{n}}^{2}\right)(\boldsymbol{\omega})$.

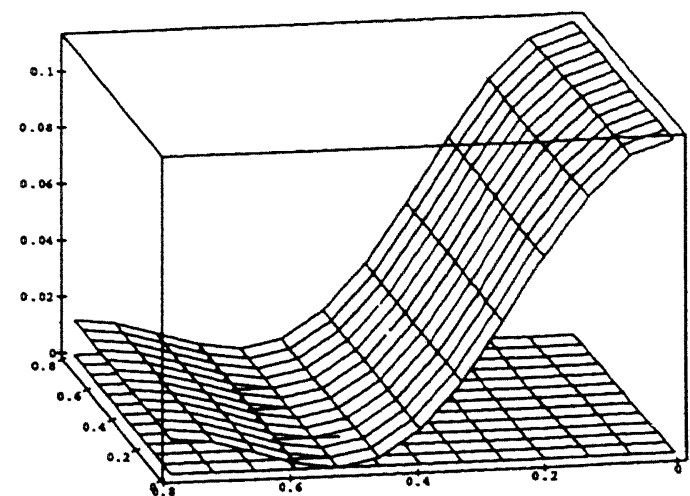

(b) The singular values of $\lim _{\varepsilon \rightarrow 0} \mathcal{F}\left(M_{\mathbf{n}}^{2}\right)(\boldsymbol{\omega})$.

Figure 4. The frequency response of the operator $\lim _{\epsilon \rightarrow 0} M_{\mathbf{n}}^{2}$, for $\mathbf{q}=(2,2)$ and $\alpha=2 / 3$.

$\max _{\epsilon}\left\|M_{\mathrm{n}}^{2}\right\| \approx 0.11$. The fact that $\left\|M_{\mathrm{n}}\right\|>1$ and $\left\|M_{\mathrm{n}}^{2}\right\| \ll 1$ shows that, in general, a W-cycle will be necessary to guarantee a good convergence rate for the algorithm.

Although only $4 \times 4$-matrices, the expressions for $\mathcal{F}\left(M_{\mathbf{n}}\right)(\boldsymbol{\omega})$ or $\mathcal{F}\left(C_{\mathbf{n}}\right)(\boldsymbol{\omega})$ are too complex to show them here explicitly. However, to understand their behaviour it is interesting to see how the matrices behave in the neighbourhood of the origin. Therefore we expand the elements of $\mathcal{F}\left(M_{n}\right)(\omega)$ in powers of $\omega$ and we show the principal terms. We see that

$$
\left[\begin{array}{cccc}
\frac{\left(-\epsilon^{2}-\sigma^{2}\right)^{2} \epsilon^{2} \sigma^{2}}{8\left(\epsilon^{2}+1\right)^{2}\left(\epsilon^{2}+\sigma^{2}\right)^{2}} \omega^{4} & \frac{\left(\epsilon^{2}+\sigma^{2}\right) \sigma^{2}\left(-\epsilon^{2}+\delta \sigma^{2}\right)}{2\left(\epsilon^{2}+1\right)^{2}\left(\epsilon^{2}+\sigma^{2}\right)^{2}} \omega & \frac{\left(-\epsilon^{2}-\sigma^{2}\right) \epsilon^{4}\left(\sigma^{2}-\delta \epsilon^{2}\right)}{2 \sigma\left(\epsilon^{2}+\sigma^{2}\right)^{2}\left(\epsilon^{2}+1\right)^{2}} \omega & \frac{\left(-\epsilon^{2}-\sigma^{2}\right) \delta}{\sigma\left(\epsilon^{2}+1\right)} \\
\frac{\left(\epsilon^{2}+\sigma^{2}\right) \sigma^{2}\left(-\epsilon^{2}+\delta \sigma^{2}\right)}{8\left(\epsilon^{2}+8\right)\left(\epsilon^{2}+\sigma^{2}\right)^{2}} \omega^{3} & \frac{\left(-\epsilon^{2}+\delta \sigma^{2}\right)^{2} \sigma^{2} \epsilon^{2}\left(2+\epsilon^{2}\right)}{4\left(\epsilon^{2}+1\right)^{2}\left(\epsilon^{2}+\sigma^{2}\right)^{2}} \omega^{2} & \frac{\left(\epsilon^{2}-\delta \sigma^{2}\right)\left(\sigma^{2}-\delta \epsilon^{2}\right) \epsilon^{2}}{\sigma\left(\epsilon^{2}+\sigma^{2}\right)^{2}\left(\epsilon^{2}+1\right)} & \frac{\left(\epsilon^{2}-\delta \sigma^{2}\right) \epsilon^{2} \delta}{2 \sigma\left(\epsilon^{2}+1\right)} \omega \\
\frac{\sigma\left(-\epsilon^{2}-\sigma^{2}\right) \epsilon^{2}\left(\sigma^{2}-\delta \epsilon^{2}\right)}{\left(8 \epsilon^{2}+8\right)\left(\epsilon^{2}+\sigma^{2}\right)^{2}} \omega^{3} & -\frac{\left(\sigma^{2}-\delta \epsilon^{2}\right)\left(-\epsilon^{2}+\delta \sigma^{2}\right) \sigma}{\left(\epsilon^{2}+\sigma^{2}\right)^{2}\left(\epsilon^{2}+1\right)} & \frac{\left(\sigma^{2}-\delta \epsilon^{2}\right)^{2}\left(1+2 \epsilon^{2}\right)}{4\left(\epsilon^{2}+1\right)^{2}\left(\epsilon^{2}+\sigma^{2}\right)^{2}} \omega^{2} & \frac{\left(\sigma^{2}-\delta \epsilon^{2}\right) \delta}{2 \epsilon^{2}\left(\epsilon^{2}+1\right)} \omega \\
\frac{\sigma\left(-\epsilon^{2}-\sigma^{2}\right) \delta}{4 \epsilon^{2}+4 \sigma^{2}} \omega^{2} & -\frac{\delta \epsilon^{2}\left(-\epsilon^{2}+\delta \sigma^{2}\right) \sigma}{\left(2 \epsilon^{2}+2 \sigma^{2}\right)\left(\epsilon^{2}+1\right)} \omega & \frac{\left(\sigma^{2}-\delta \epsilon^{2}\right) \delta}{\left(2 \epsilon^{2}+2 \sigma^{2}\right)\left(\epsilon^{2}+1\right)} \omega & \delta^{2}
\end{array}\right]
$$

$$
\mathcal{F}\left(M_{\mathbf{n}}\right)(\omega) \approx
$$

where $\delta=2 \alpha-1$ is the damping parameter, and we denote $\omega=\omega_{1}$ and $\omega_{2}=\sigma \omega$.

We notice that a singularity is present at the origin. The $\operatorname{limit}_{\lim _{\omega \rightarrow 0}} \mathcal{F}\left(M_{\mathbf{n}}\right)(\omega)$ depends on the ratio $\sigma=\omega_{2} / \omega_{1}$. This is the reason why the eigen- and singular values are missing at $\boldsymbol{\omega}=\mathbf{o}$ in figures 1 and 2 .

\section{Summary}

In this paper we describe a multigrid algorithm for second order elliptic equations in three dimensions. We assume finite-volume discretisation. For the approximation we use piecewise constant basis functions, that are the tensor product generalisation of the one-dimensional case. Using a family of uniform 
grids, each member with a different size of support in the different coordinate directions, we obtain a hierarchy of approximations. The corresponding set of function spaces can be interpreted in terms of wavelet terminology as a three-dimensional multiresolution analysis. Following the idea of sparse grids, a selection of degrees of freedom is made, that gives a high accuracy for a relatively small number of degrees of freedom, provided that a certain smoothness requirement is satisfied.

A multigrid algorithm of additive Schwarz type is now constructed for the solution of the discrete system, and its convergence is analysed by Fourier analysis. For this purpose the necessary tools are developed for the Fourier analysis of the box-grid functions.

From the analysis of which the results are summarized in figures 1-4, we conclude that, with simple damped Jacobi iteration as a smoother, the spectral radius of the multigrid iteration matrix is less than 1, uniformly in the cell aspect ratio. In fact, for $\epsilon$ the cell aspect ratio, we find $\max _{\epsilon} \rho\left(M_{\mathrm{n}}\right) \approx 0.33$.

The spectral norm can be larger than one. We find $\max _{\epsilon}\left\|M_{\mathbf{n}}\right\| \approx 5.0$. This may indicate that a V-cycle type algorithm will not converge. However, it appears that $\max _{\epsilon}\left\|M_{\mathrm{n}}^{2}\right\| \approx 0.11$. This shows that, in general, a W-cycle will be necessary to guarantee a good convergence rate for the algorithm.

\section{References}

[1] R.N. Bracewell, The Fourier Transform and its Applications (McGraw-Hill, 1986).

[2] I. Daubechies, Ten Lectures on Wavelets, vol. 61 of CBMS-NSF regional conference series in applied mathematics (SIAM, 1992).

[3] M. Griebel, M. Schneider and C. Zenger, A combination technique for the solution of sparse grid problems, Technical Report TUM-I 9038, SFB 342/19/90A, TU München (1990).

[4] P.W. Hemker, Remarks on sparse-grid finite-volume multigrid, Technical Report NM-R9421, CWI, Amsterdam (1994).

[5] P.W. Hemker and P.M. de Zeeuw, BASIS3: A data structure for 3-dimensional sparse grids, Technical Report NM-R9321, CWI, Amsterdam (1993).

[6] W. Mulder, A high-resolution Euler solver based on multigrid semi-coarsening, and defect correction, J. Comp. Phys. 100 (1992) 91-104.

[7] W.A. Mulder, A new multigrid approach to convection problems, J. Comp. Phys. 17 (1989) 303323.

[8] N.H. Naik and J. Van Rosendale, The improved robustness of multigrid elliptic solvers based on multiple semicoarsened grids, Technical Report No. 91-70, ICASE (1991).

[9] C. Zenger, Sparse grids, in: Parallel Algorithms for PDE, Proc. 6th GAMM Seminar, ed. W. Hackbusch, Kiel 1990 (Vieweg, Braunschweig, 1991) pp. 241-251. 